\title{
Genetic Analysis of the Drosophila Ellipsoid Body Neuropil: Organization and Development of the Central Complex
}

\author{
Susan C. P. Renn, ${ }^{1}$ J. Douglas Armstrong, ${ }^{2, *}$ Mingyao ${ }^{\text {Yang, }}{ }^{2, *}{ }^{2}$ Zongsheng Wang, ${ }^{2}$ \\ Xin An, ${ }^{2}$ Kim Kaiser, ${ }^{2}$ Paul H. Taghert ${ }^{1}$ \\ ${ }^{1}$ Department of Anatomy and Neurobiology, Washington University School of Medicine, \\ 660 S. Euclid Avenue, St. Louis, Missouri 63110 \\ 2 Division of Molecular Genetics, Anderson College, University of Glasgow, \\ 56 Dumbarton Road, Glasgow G11 6NU, United Kingdom
}

Received 2 December 1998; accepted 24 March 1999

\begin{abstract}
The central complex is an important center for higher-order brain function in insects. It is an intricate neuropil composed of four substructures. Each substructure contains repeated neuronal elements which are connected by processes such that topography is maintained. Although the neuronal architecture has been described in several insects and the behavioral role investigated in various experiments, the exact function of this neuropil has proven elusive. To describe the architecture of the central complex, we study 15 enhancer-trap lines that label various ellipsoid body neuron types. We find evidence for restriction of gene expression that is correlated with specific neuronal types: such correlations suggest functional classifications as
\end{abstract}

well. We show that some enhancer-trap patterns reveal a single ellipsoid body neuron type, while others label multiple types. We describe the development of the ellipsoid body neuropil in wild-type animals and propose developmental mechanisms based on animals displaying structural mutations of this neuropil. The experiments performed here demonstrate the degree of resolution possible from the analysis of enhancer-trap lines and form a useful library of tools for future structure/function studies of the ellipsoid body. () 1999 John Wiley \& Sons, Inc. J Neurobiol 41: 189-207, 1999

Keywords: enhancer-trap; central complex; ellipsoid body; neuropil; structural mutant
The central complex (cc) is one of the most striking features of the adult insect brain. Unlike other principal neural centers, such as the mushroom bodies (mb), antennal lobes, and optics lobes, the cc spans the sagital midline and is symmetrically organized (Power, 1943). The disposition of the cc explains early suggestions that the structure plays a role in

\footnotetext{
* These authors contributed equally to this work.

Correspondence to: $\mathrm{P}$. Taghert

Contract grant sponsor: UK BBSRC

Contract grant sponsor: Wellcome Trust

Contract grant sponsor: NIH; contract grant number: NS-21749

(C) 1999 John Wiley \& Sons, Inc. CCC 0022-3034/99/020189-19
}

coordination between brain hemispheres (reviewed by Homberg, 1987). The cc neuropil can be identified among insects from diverse habitats (Schistocerca: Williams, 1975; Muller et al., 1997; Musca: Strausfeld, 1976; Apis: Homberg, 1985; Schurmann and Klemm, 1984; Calliphora: Lundquist et al., 1994; Periplaneta: Mizunami, 1995; Acheta: Schildberger, 1983; Tenebrio: Wegerhoff and Breidbach, 1992; Wegerhoff et al., 1996). This suggests that the structure may subserve functions shared among broadly divergent insect species.

While the cc has attracted attention as a principal region of higher-brain organization, evidence supporting a specific behavioral role has proven elusive (for 


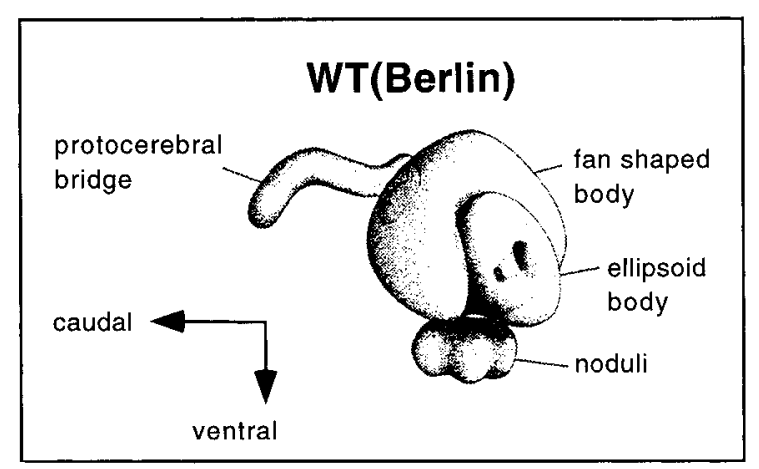

Figure 1 Schematic drawing of the central complex. Reproduced with permission from Strauss and Heisenberg (1993).

review, see Homberg, 1987, Heisenberg, 1994). Classic structural analysis (Strausfeld, 1976; Hanesch et al., 1989), stimulus and ablation studies (Wadepuhl and Huber, 1979; Graham, 1979a,b), genetic manipulations (Strauss and Heisenberg, 1993; Strauss et al., 1992; Bouhouche et al., 1993; Illius et al., 1994), and activity-dependent labeling (Bausenwein et al., 1994) support the hypothesis that the cc neuropil is a site of behavioral control. Abnormal walking behavior, and flight behavior (Illius et al., 1994) are correlated with genetic mutations that disrupt the cc structure. Interestingly, only specific components of the behavior are disrupted in each case; for example, basic leg coordination of walking is normal, whereas speed, activity, and turning are affected (Strauss and Heisenberg, 1993; Strauss et al., 1992). In wild-type flies, it has been shown that walking and flight causes an increase in cc neuropil activity. Furthermore 2-deoxyglucose labeling demonstrates that different neuropil regions are activated by each behavior (Bausenwein et al., 1994). These results suggest that the $\mathrm{cc}$ is an important site for fine-tuning of behavior. In addition to its role in locomotor behavior, there is evidence that the cc may play a role in olfactory associative learning and memory (Heisenberg, 1989; Bouhouche et al., 1993).

The cc is composed of four interconnected neuropils: the protocerebral bridge (pb), the fan shaped body (fb), the ellipsoid body (eb), and the noduli (no) (Power, 1943) (Fig. 1). The ventral bodies (vbo) (or lateral accessory region), and the lateral triangles (ltr) are closely associated structures, predominantly formed by the processes of extrinsic neurons as described by Hanesch et al. (1989). A detailed study of some 1200 Golgi-impregnated adult brains by Hanesch et al. (1989) revealed approximately 30 different neuronal types based upon morphology and topography. The majority of these classes were categorized as being either large-field or small-field neurons. Largefield neurons typically arborize within one or more tangential layers of a single cc subunit (e.g. within layers of the $\mathrm{fb}$ or entire rings of the eb) and provide connectivity to one or more accessory structures or non-cc areas. The majority of small-field neurons interconnect small subdivisions within one or more cc subunit. For example, intrinsic small-field neurons divide the $\mathrm{pb}$ and $\mathrm{fb}$ as regular columnar elements, and the eb into radial elements. Small-field neurons interconnect elements of different substructures, and some small-field neurons also project to the accessory areas or other brain regions. Golgi studies of Hanesch et al. (1989) describe the complex three-dimensional structure of the Drosophila cc as similar to that of Schistocerca (Williams, 1975). The cc displays a high degree of intrinsic, topographic order and participates with many functionally diverse and widely situated brain centers.

To further the functional analysis of the cc, we performed three sets of anatomical and developmental studies focusing on one of its principal regions, the eb, using enhancer-trap technology. These methods represent a valuable source of type-specific neuronal markers that provide anatomical details of cellular phenotype not afforded by conventional histological techniques (Bier et al., 1989; Bellen et al., 1989). The genetic basis of enhancer-trap techniques indicates not just anatomical relations, but potentially developmental and/or functional relations as well. The P $\{$ GAL4 $\}$ enhancer-trap system (Brand and Perrimon, 1993; Brand and Dormand, 1995) is especially effective in this regard, as it provides a simple means of examining brain anatomy at high cellular resolution and at various stages of development (Armstrong and Kaiser, 1997, Armstrong et al., 1998).

The first set of experiments interpreted a suite of P $\{$ GAL4 $\}$ enhancer-trap lines that mark subsets of eb neurons, in patterns that are reminiscent of previously described eb cell morphologies. We focused specifically on five $P\{G A L 4\}$ lines that illustrate the diversity of large-field neurons projecting to the eb. We used double-labeling studies with a $\mathrm{P}\{$ lac $Z\}$ enhancer-trap line to describe arborization boundaries for eb neurons of different morphologies. While some lines represent single eb neuronal types, others mark neurons belonging to multiple types. These correlated patterns of reporter gene expression suggest a relation between gene expression and neuronal morphology. The second set of experiments addressed the developmental origins of the eb. There is little information concerning the elaboration of the cc during Drosophila metamorphosis; however, one study revealed a pb-like structure in the white pupal stage (Schneider 
Table 1 eb Staining Patterns Observed in 16 Independent P-element Lines

\begin{tabular}{|c|c|c|c|c|}
\hline Line & Location & Figure No. & Neuron Type & \\
\hline $\mathrm{c} 161$ & $66 \mathrm{~A}$ & $2(\mathrm{~A}-\mathrm{C}), 3(\mathrm{~A})$ & small field & \\
\hline $78 \mathrm{Y}$ & $84 \mathrm{D}$ & 2(D,E), 3(B) & small field & \\
\hline $007 \mathrm{Y}$ & ND & $2(\mathrm{~F}, \mathrm{G})$ & small field & \\
\hline c107 & $19 \mathrm{~F}$ & $2(\mathrm{H}, \mathrm{I})$ & small field & \\
\hline $64 \mathrm{Y}$ & $70 \mathrm{~B}$ & $4(\mathrm{~A})$ & undesignated R-type & \\
\hline $\mathrm{c} 561 / \mathrm{c} 105$ & $12 \mathrm{~F}$ & 4(B), 6(A,B), 9(C) & R1 & \\
\hline $198 Y$ & ND & $4(\mathrm{C})$ & $\mathrm{R} 1$ & \\
\hline $189 \mathrm{Y}$ & $24 \mathrm{~A}$ & 4(D), 6(C,D) & R3 & $\mathrm{mb}$ \\
\hline $\mathrm{c} 481$ & $18 \mathrm{~A}$ & $4(E)$ & R3 & \\
\hline c115 & $77 \mathrm{~A}$ & $4(F)$ & $\mathrm{R} 2, \mathrm{R} 4 \mathrm{~m}$ & $\mathrm{mb}$ \\
\hline c346 & ND & $4(\mathrm{G})$ & $\mathrm{R} 2, \mathrm{R} 4 \mathrm{~m}$ & \\
\hline $93 \mathrm{Y}$ & ND & $4(\mathrm{H})$ & undesignated R-type & \\
\hline c819/c547 & $93 \mathrm{C}$ & $4(\mathrm{I}), 7(\mathrm{C}), 9(\mathrm{~B})$ & $\mathrm{R} 2, \mathrm{R} 4 \mathrm{~m}$ & \\
\hline $\mathrm{c} 42$ & ND & $4(J), 8$ & $\mathrm{R} 2, \mathrm{R} 4 \mathrm{~m}$ & $\mathrm{Fm} 2$ \\
\hline $\mathrm{c} 232 / \mathrm{c} 507$ & 100B & $4(\mathrm{~K}), 7(\mathrm{~A}, \mathrm{~B}), 9(\mathrm{~A})$ & $\mathrm{R} 3, \mathrm{R} 4 \mathrm{~d}$ & \\
\hline pWF22-6 & $83 \mathrm{E}$ & $5-9$ & $\mathrm{R} 4 \mathrm{~d}$ & $\mathrm{mb}$ \\
\hline
\end{tabular}

The location of the P-element insertion is given for those lines for which this information is available (ND = not determined) and the presumed cell type(s) which constitute the eb staining pattern is noted.

et al., 1993b). Using the suite of lines that mark adult eb neurons, we visualized their development. Finally, we re-examined enhancer-trap expression patterns in the context of cc structural mutants (CX) (Strauss and Heisenberg, 1993). CX mutants exhibit various structural deformities of the cc and display correlated motor control deficits (Strauss and Heisenberg 1993; Bouhouche et al., 1993; Strauss et al., 1992; Illius et al., 1994). The enhancer-trap approach provides a means to visualize mutant brain structure with high resolution, and we used it as a means to evaluate the presence of specific neuronal types and the structural integrity of these disorganized neuropils.

\section{MATERIALS AND METHODS}

\section{Drosophila Strains}

This study surveyed enhancer-trap lines that can be used as markers for the eb structure (Table 1). The P $\{$ GAL4 $\}$ enhancer-trap lines were selected from a screen of $\sim 1400$ lines by Yang et al. (1995). The secondary reporters for GAL4 activity were UAS-lacZ (ULZ 4.1.2), UAS-Tetanus light chain (TeTXLC) (IMPTNT-VA) (Sweeney et al., 1995), and UAS-GFP-F1/L (Yeh et al., 1995). There was no detectable reporter gene expression in the absence of a P $\{$ GAL4 $\}$ element. The enhancer line pWF22-6 is not in the GAL4 series: it contains a direct lacZ reporter construct in a Casper-based vector including a minimal promoter from the hs43 gene and a 256-bp fragment of the $d F M R F a$ neuropeptide gene promoter (bases -418 to $-162 \mathrm{bp}$ ). This fragment produces expression in some eb neurons in a small fraction of independent transformant lines (M. Roberts,
S. C. P. Renn, and P. Taghert, unpublished observations). The eb expression in pWF22-6 animals is therefore likely due to an undefined interaction between the insertion site and the $A F M R F a$ enhancer sequences. The cc structural mutants $c b d^{K S 96}, c e b^{849}, c c b^{K S 145}, c e x^{K S 181}, e b o^{K S 263}$, and $m b d^{K S 65}$ were generated by ethylmethane sulphonate (EMS) mutagenesis of the X chromosome (Strauss and Heisenberg 1993; Heisenberg et al., 1985).

\section{X-gal Staining}

Histology was performed on adult flies of age 4-14 days. All flies were anesthetized with $\mathrm{CO}_{2}$ prior to histological preparation. Then, $12-\mu \mathrm{m}$ frontal cryostat sections of adult heads were fixed on glass slides for $15 \mathrm{~min}$ in phosphate buffered saline (PBS) containing 2\% glutaraldehyde. After washing in PBS $(2 \times 15 \mathrm{~min}$.), the sections were stained in the dark for $2-4 \mathrm{~h}$ at $37^{\circ} \mathrm{C}$ in a moist box. The staining solution was $0.2 \% \mathrm{X}$-galactosidase (X-gal) [diluted from an $8 \%$ stock solution in dimethylsulfoxide (DMSO)] in prewarmed FeNaP buffer. FeNaP buffer is $10 \mathrm{mM}$ $\mathrm{NaH}_{2} \mathrm{PO}_{4} \cdot \mathrm{H}_{2} \mathrm{O}, 10 \mathrm{~m} M \mathrm{Na}_{2} \mathrm{HPO}_{4} \cdot 2 \mathrm{H}_{2} \mathrm{O}, 150 \mathrm{~m} M \mathrm{NaCl}, 1$ $\mathrm{m} M \mathrm{MgCl}_{2} \cdot 6 \mathrm{H}_{2} \mathrm{O}, 3.1 \mathrm{~m} M \mathrm{~K}_{4}\left(\mathrm{Fe}^{2+} \mathrm{CN}\right)_{6}, 3.1 \mathrm{~m} M$ $\mathrm{K}_{3}\left(\mathrm{Fe}^{3+} \mathrm{CN}\right)_{6}, 0.3 \%$ Triton $\mathrm{X}-100, \mathrm{pH}$ 7.4. The staining solution was made fresh each day and kept dark. After postwashing in PBS for $10 \mathrm{~min}$ and dehydration though graded ethanol, the preparations were mounted in glycerol/ gelatin (Sigma). Images were scanned on a Zeiss Axiophot microscope using a Kontron ProgRes 3008 camera and a $\times 160.5$ NA lens (and a $\times 0.5$ magnification $C$-mount).

\section{Immunohistochemistry}

Immunohistochemistry was performed on whole-mount tissue of 2- to 5-day-old adult, pupal, and third larval instar 
CNS as described by Schneider et al. (1993b). Briefly, dissections were performed in a simple saline $(180 \mathrm{mM}$ $\mathrm{KCl}, 50 \mathrm{~m} M \mathrm{NaCl}, 2 \mathrm{~m} M \mathrm{CaCl}_{2}, 10 \mathrm{~m} M$ Tris, $\mathrm{pH}$ 7.2) and the tissues were fixed with $4 \%$ paraformaldehyde in PBS overnight at $4{ }^{\circ} \mathrm{C}$. All preblocking and antibody incubations were performed in PBS containing $0.3 \%$ Triton X-100, $10 \%$ normal goat serum, $1 \mathrm{mg} / \mathrm{mL}$ bovine serum albumin (BSA), and $0.01 \%$ sodium azide. Primary antibody incubations proceeded overnight at $4^{\circ} \mathrm{C}$; all rinses, and the 2-h secondary antibody incubations were performed at room temperature, all with constant agitation. The $\beta$-gal expression of the pWF22-6 element or GAL4-driven UAS-lacZ was vi- sualized using either mouse (Promega Corp.; diluted $1: 1000)$ or rabbit anti- $\beta$-gal antiserum (Cappel; diluted 1:2000) and either Texas red-, fluorescein isothiocyanate (FITC)-, or horseradish peroxidase (HRP)-conjugated secondary antibodies (Jackson Lab, diluted 1:200; or Molecular Probes, diluted 1:500). For double-labeling experiments, the pWF22-6 line was crossed with both a P $\{$ GAL4 $\}$ enhancertrap line of interest and either UAS-GFP or UAS-(inactive)TeTxLC (Sweeney et al., 1995). Inactive TeTXLC was chosen for compatibility with anti- $\beta$ gal immuno-staining and for its robust staining of axons. It was visualized with a monoclonal anti-TeTxLC at a dilution of 1:10,000 and
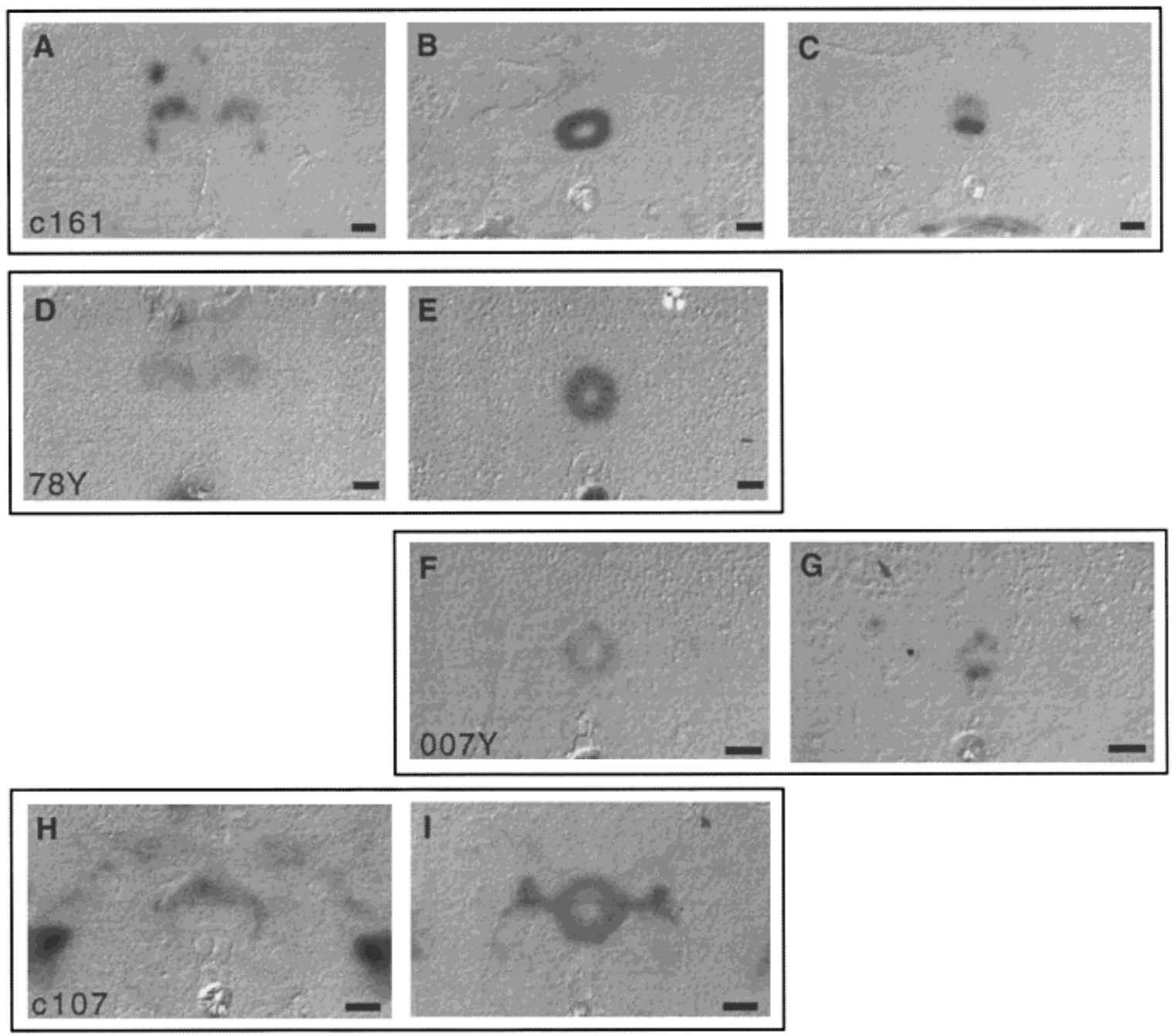

Figure 2 X-Gal-stained frontal cryostat head sections from four independent $P\{G A L 4\}$ lines that stain small-field neurons projecting to the eb. Boxes surrounding panels indicate images derived from a single tissue. Numbers in the bottom left of each box (e.g., c161 in the top box) indicate names of lines. Scale bars indicate $20 \mu \mathrm{m}$. Lines c161, 78Y, and 007Y stain small-field neurons which, to varying degrees, connect the eb (B,E,F) with the pb and no; X-Gal stain clearly reveals the ventral no in lines c161 and $007 \mathrm{Y}(\mathrm{C}, \mathrm{G})$, but staining of the dorsal no in each line is obscured by eb axons (B,E,F). The pb is stained robustly in lines c161 (A) and 78Y (D), whereas in line 007Y the $\mathrm{pb}$ is stained weakly (not shown). Line c107 stained another type of small field neuron which connected the eb (I) and the $\mathrm{fb}(\mathrm{H})$. 
FITC-labeled anti-mouse secondary antibodies (Jackson Lab, diluted 1:200).

\section{Developmental Staging}

To study eb development, we collected and determined the sex of animals within $30 \mathrm{~min}$ of puparium formation. They were then reared at $25^{\circ} \mathrm{C}$ until ready for dissection. Both male and female pupae were dissected at 2- to 4-h intervals. Dissection times were accurate to $\pm 5 \mathrm{~min}$ and collection times accurate to $\pm 30 \mathrm{~min}$. Stages are reported as hours after puparium formation (APF). The time from pupariation to eclosion was approximately $120 \mathrm{APF}$ under these conditions.

\section{Confocal Microscopy}

Confocal microscopy was performed using two different systems. Confocal images were scanned on a Molecular Dynamics confocal microscope using excitation $(480 \mathrm{~nm})$ and detection $(530 \pm 15 \mathrm{~nm})$ filters for fluorescein-based secondary antibodies and excitation $(568 \mathrm{~nm})$ and detection $(620 \pm 20 \mathrm{~nm})$ filters for Texas red-based secondary antibodies. Alternatively, images were scanned on an Olympus confocal microscope using excitation $(488 \mathrm{~nm})$ and detection $(510-550 \mathrm{~nm})$ filters for fluorescein and excitation (568 $\mathrm{nm})$ and a detection $(610 \mathrm{~nm})$ filter for Cy3-based secondary antibodies. Tissues were studied at $\times 20, \times 40$, and $\times 60$. Three-dimensional reconstructions were performed using the programs ImageSpace 3.1 (Molecular Dynamics) and
Fluoview 1.1 (Olympus). Color images and montages were constructed using NIH Image and Adobe Photoshop.

\section{Chromosome Cytology}

The procedure for in situ hybridization to polytene chromosomes was essentially as described by Pardue (1986). Briefly, larvae were grown on rich larval medium and third-instar larvae were chosen for chromosome squashes. pBluescript DNA was labeled with Bio-16-dUTP by nicktranslation and hybridization was detected using 3,3'-diaminobenzimidine (Sigma). After hybridization, the slides were stained with Giemsa and mounted using DPX (Fluka).

\section{RESULTS}

\section{P\{GAL4\} Enhancer-Trap Expression in the Ellipsoid Body}

From a P $\{$ GAL4 $\}$ mutagenesis, a total of 1400 P $\{$ GAL4 $\}$ lines were isolated and screened for GAL4directed lacZ expression by histochemical staining of cryostat frontal sections of the Drosophila head (Yang et al., 1995; Armstrong and Kaiser, 1997). Fifteen lines were selected on the basis of eb staining (Fig. 2; 4 and 6). For each of the lines described, Table 1 indicates the figures that illustrate the staining patterns and the neuron type presumed to make up that pattern.
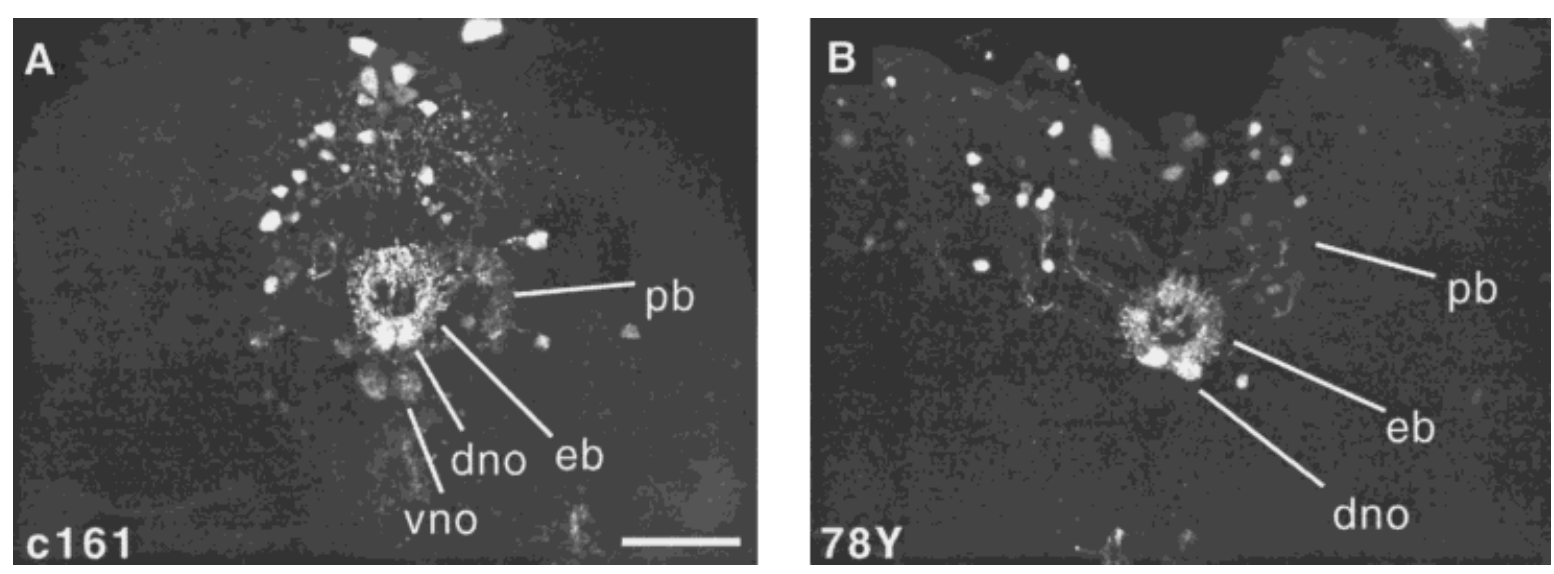

Figure 3 Confocal microscopy of adult brains showing $\mathrm{P}\{\mathrm{GAL} 4\}$ directed expression of lacZ in two lines that reveal small field neurons arborizing in the pb, eb, and no. (A) Line c161 stained cell bodies clustered in the medial protocerebrum which send projections to the glomeruli of the pb; axons also arborize throughout the eb and both the dorsal (dno) and ventral (vno) regions of the no. This pattern is highly reminiscent of pb-eb-no neurons described by Hanesch et al. (1989). There is also expression in a network of fibers just dorsal to the anterior medial protocerebrum which appears to be connected to the cc neurons. (B) Line 78Y stained a cluster of cc neurons located in a more lateral position than that of line c161 (some overlap cannot be discounted). They project to the pb and from there progress to the eb, where they innervate the inner/midregion of the ring from the canal outward. The no staining in line $78 \mathrm{Y}$ is restricted to the dorsal regions (dno), and in Figure 2 (E) was obscured by eb staining. Scale bar $=50 \mu \mathrm{m}$. 
Staining was not exclusive to the eb nor to the nervous system. Non-cc staining aspects are described below. Chromosomal locations were obtained for most lines (Table 1) and reveal that, apart from just three pairs, all insertion sites were different. For each insertion pair, $\mathrm{P}\{\mathrm{GAL} 4\}$-directed lacZ expression patterns were indistinguishable.

Among the selected P $\{$ GAL4 $\}$ lines, we found patterns reminiscent of several of the major eb morphological types revealed by Golgi impregnation (Hanesch et al., 1989). The eb is organized in a concentric pattern of 12-16 glomeruli around its circumference. It is also divisible along the anterior- posterior axis into two principal disks. Small-field neurons interconnect the anterior and posterior layers of the eb, and connect the eb to other cc substructures. Among the major contributions to the eb are the projections of large-field neurons, termed $\mathrm{R}$ neurons, that arborize as a ring mainly in the anterior disk (Hanesch et al., 1989).

\section{Staining Patterns That Include Small-Field Neurons of the eb}

Hanesch et al. (1989) described 10 types of small field neurons that arborized within the eb. Four of the
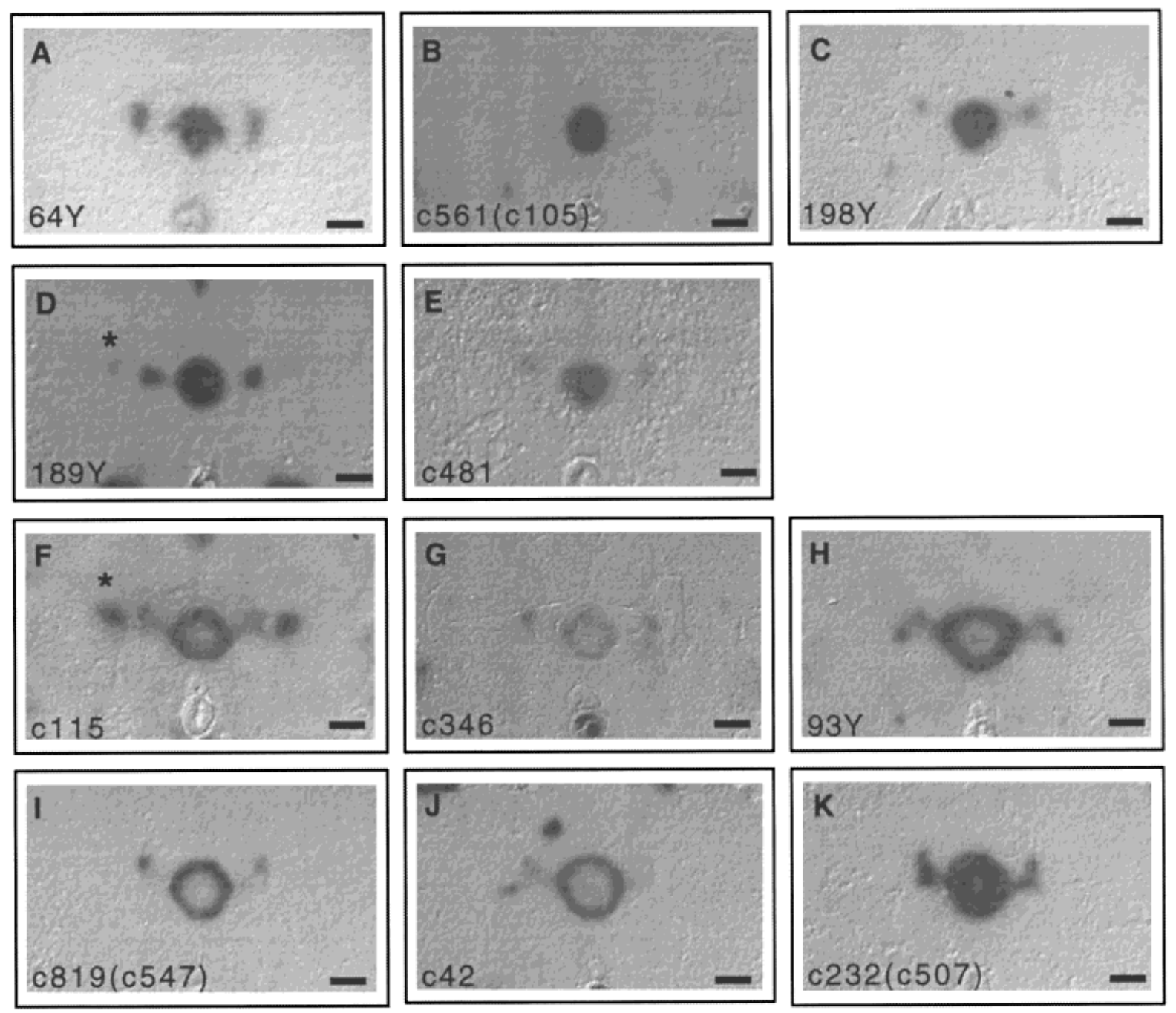

Figure $4 \mathrm{X}$-Gal-stained frontal cryostat head sections from 11 independent $\mathrm{P}\{\mathrm{GAL} 4\}$ lines that predominantly stain large-field neurons of the eb. Stock numbers in parentheses indicate lines with a similar P-insertion site and a comparable staining pattern. (A-K) Range of arborization domains seen in lines that stain R-type neuronal processes. Lines that stained inner domains are grouped in upper panels (A-E), and those that stained outer domains, in lower panels (G-L). Some lines clearly displayed staining in multiple ring domains [e.g., (L)]. The mb pedunculus stained in lines c115 and $189 \mathrm{Y}$ is indicated with an asterisk. Scale bars $=20 \mu \mathrm{m}$. 
eb-staining P $\{$ GAL4 $\}$ lines displayed patterns which suggested small-field neuron representation (Table 1). Patterns in lines c161, 78Y, and c007 each suggested the pb-eb-no type of small-field neuron [Fig. 2(A-G)]. This was most clearly seen for line c161 [Fig. 2(AC)]. Staining of the dorsal no was not easily visualized with X-gal staining owing to the overlying eb axons [c161, Fig. 2(B), and 78Y, Fig. 2(E)]. In each line containing small-field neurons, the different $\mathrm{cc}$ substructures showed different relative levels of X-gal staining. Line $007 \mathrm{Y}$ [Fig. 2(F,G)] robustly stained the eb [Fig. 2(F)] and the no [Fig. 2(F)], while the pb stained only faintly (not shown.) The pattern in line c107 resembled an fb-eb type connecting the two substructures [Fig. 2(H,I)]. In most cases, the large number of stained neurons precluded accurate tracing of individual axons, and so prevented assigning an absolute identity to particular small-field neurons. For example, the putative pb-eb-no type in line c161 may instead have reflected coordinate expression by multiple eb small-field types, e.g. pb-eb-eb and pb-no. Consistent with the supposition that different cell types participate in staining patterns for the different small-field neuron enhancer-trap lines, confocal analysis revealed differences between them [Fig. 3(A,B)]. For example, c161 stained several cell bodies with medial locations, while cell bodies in line $78 \mathrm{Y}$ had more lateral placement. Also, fasciculated processes traveling between certain $\mathrm{pb}$ glomeruli and the eb canal were a feature specific to line $78 \mathrm{Y}$.

\section{Staining Patterns That Include Large-Field $\boldsymbol{R}$ Neurons of the eb}

Other lines displayed staining indicative of $\mathrm{R}$ neuron expression. The primary neurites of $\mathrm{R}$ neurons run in the prominent RF tract. Near the ltr, the neurites of the $\mathrm{R}$ neurons split to send dendrites into the 1 tr, and axons into the eb (Hanesch et al., 1989). R neurons have been classified into four types (R1-4) based on the position at which the axon enters the eb neuropil, and based on the relative radius of its concentric arborization. Figure 6 shows various eb staining patterns to illustrate the diversity of fully tangential (i.e., circular) R neuron arborization patterns. Side-by-side comparisons suggest that most, if not all, of the concentric domains (from proximal to distal) of the eb are encompassed by this suite of enhancer-trap expression patterns. However, we did not find any single pattern that included the entire $\mathrm{R}$ neuron population. We conducted a further detailed analysis using five selected lines which presented a comprehensive and representative collection of the $\mathrm{R}$ neuron types.

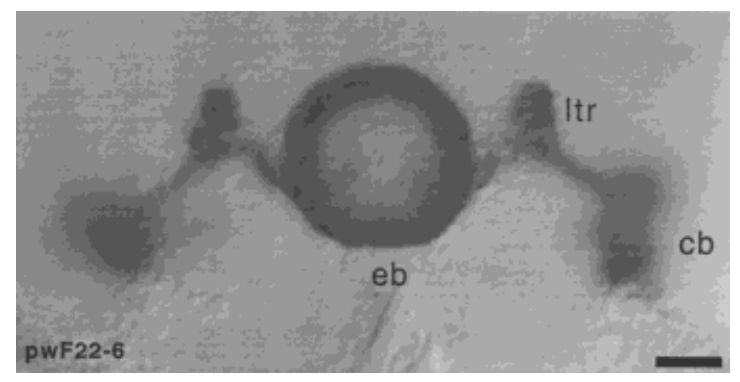

Figure 5 Histochemical staining of R4-type eb neurons in the pWF22-6 direct lacZ P line. The axons enter the neuropil at the periphery of the ring and demarcate its perimeter. The cell bodies (cb) are located on the anterior surface of the brain (out of focus); the processes arborize in the ltr and before reaching the eb. These stained neurons represent only a portion of all R4 type neurons (see text). Scale bar $=20 \mu \mathrm{m}$.

\section{Relating Enhancer-Trap Expression Patterns to Morphologically Defined R Neuron Classes}

To examine the relationship between enhancer-trap expression patterns and morphological types previously defined by Golgi studies, we used an additional marker: a lacZ-bearing P-element line called pWF22-6, whose expression pattern includes strong staining of $\mathrm{R}$ neurons that arborize at the most distal radial zone of the eb (Fig. 5). As described by Hanesch et al. (1989), R1-3 neurons project to the eb via the eb canal and arborize outwardly from that point; axons of the $\mathrm{R} 4$ neurons reach the eb at its distal radial circumference, and they arborize in the outer ring. R1-3 neurons are further distinguished by their arborization zones within the eb. R1 axons arborize in an inner ring, while $\mathrm{R} 2$ axons arborize in an outer ring (similar to R4), and R3 axons arborize throughout both inner and midrings. Axons of R2, R3, and R4 neurons are restricted to the anterior eb, while R1 axons extend to the posterior layer. Neurons equivalent to each of the four previously described $\mathrm{R}$ neurons are represented in the various enhancer patterns.

\section{A Few Lines Predominantly Labeled a Single $R$ Neuron Type.}

Line pWF22-6 labeled only an R4-type arborization, entering at and restricted to the outer perimeter of the eb (Figs. 5 and, the red channel in Figs. 6-11 and 13). Line c561/c105 labeled only R1-type neurons that entered at and were restricted to the inner portion of the eb [Figs. 4(B) and 6(A,B) green]. Line c561/c105 stained 6-10 cell bodies lateral to the $\mathrm{cc}$ in each hemisphere from which arise neurites that project to 
the $\operatorname{ltr}$ [Fig. 6(A)]. R4 axons of pwF22-6 were restricted to the anterior disk, while the R1 axons of c561/c105 extended into the posterior disk of the eb [Fig. 6(B)]. A similar eb arborization pattern was seen in line 198Y [Fig. 4(C)]. Axons of lines 189Y [Figs. $4(\mathrm{D})$ and $6(\mathrm{C}, \mathrm{D})]$ entered the eb via the canal and arborized throughout much of the anterior eb layer reminiscent of R3 morphology. A similar eb arborization pattern was seen in line c481 [Fig. 4(E)]. In cross-section, a few axons in line $189 \mathrm{Y}$ extended into the posterior layer [Fig. 6(D)] suggestive of costaining of another neuron class, possibly R1-type or small-field neurons.

\section{Several Lines Labeled Multiple $\boldsymbol{R}$ Neuron Types}

Line c232/c507 labeled two morphological types of R-neurons [Figs. 4(K) and 7(A,B)]. In addition to double labeling with the majority of the pWF22-6positive R4 neurons, line c232/c507 showed expression in R3 neurons. The latter appeared similar to R3 neurons labeled in line 189Y. Line c232/c507 expression was highly eb specific in that no other neurons in the brain were visible. Lines c819/c547 [Figs. 4(I) and 7(C)] and c42 [Figs. 4(J) and 8] labeled R2- and R4-type neurons. Extensive arborization was visible

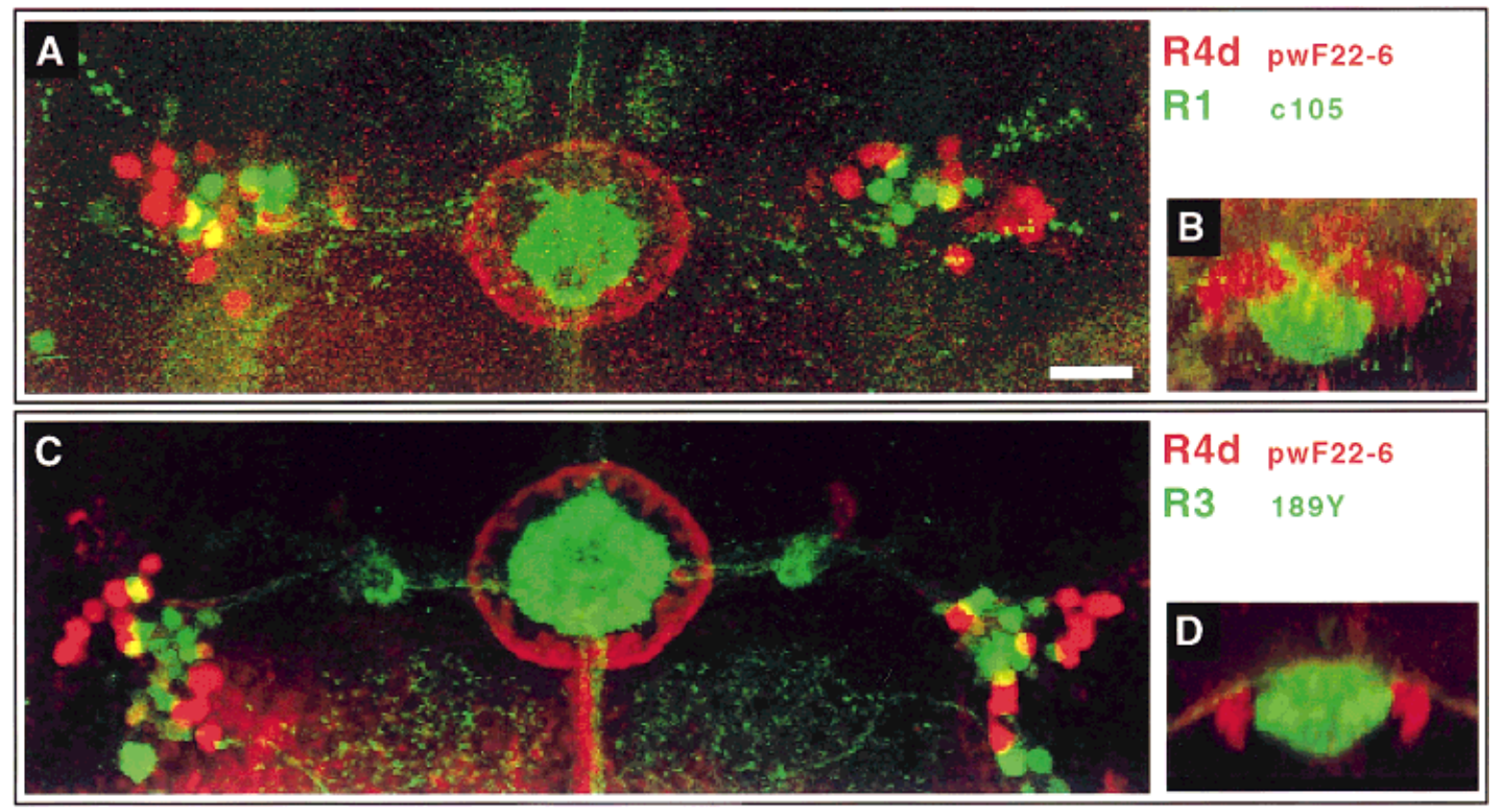

Figure 6
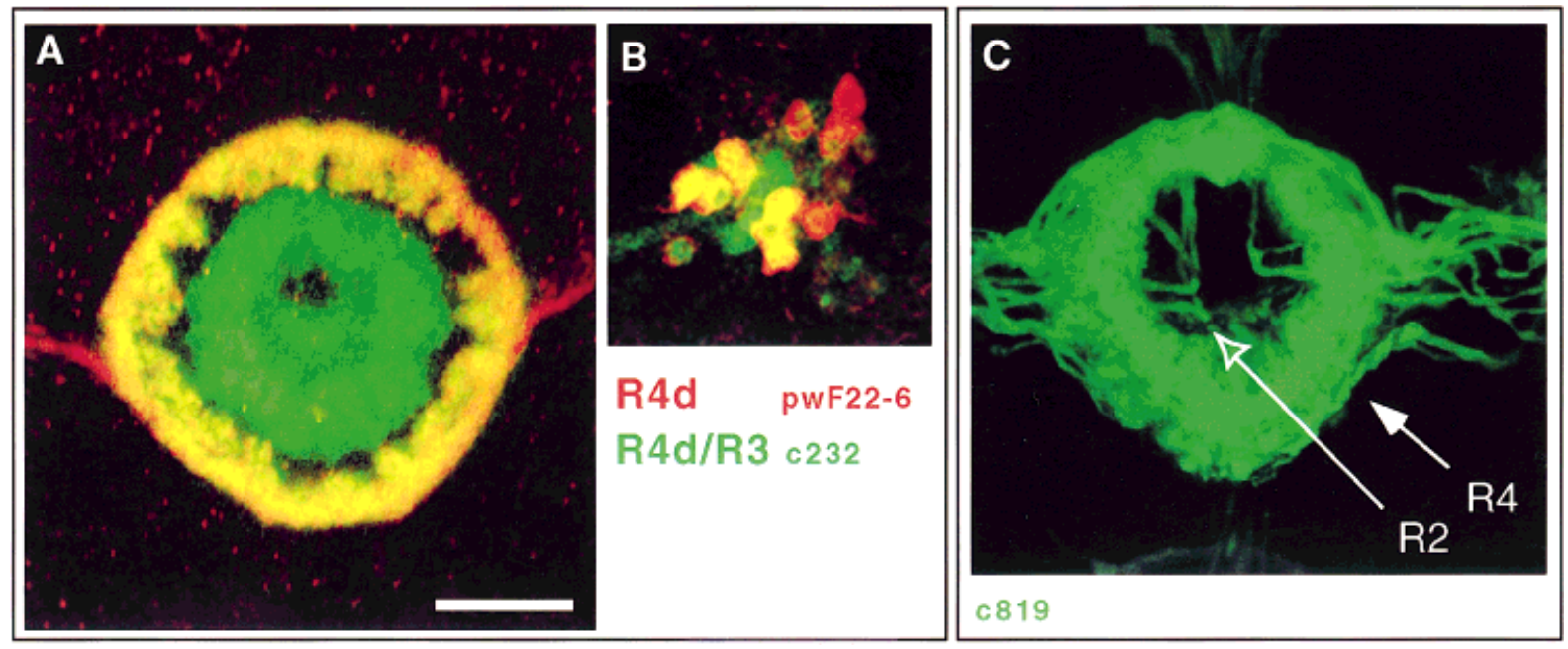

Figure 7 
in the outer zone, with axons entering the eb neuropil both peripherally (R4-like) and centrally via the canal (R2-like) [shown for c819 in Fig. 7(C)].

\section{Several Lines Revealed Subdivisions within the R4 Class}

Although pWF22-6 and several of the P $\{$ GAL $\} 4$ lines featured R4-type neurons, the eb patterns were not equivalent. The pWF22-6 arbors, which were also labeled in line c232/c507 [Fig. 7(A,B)], occupied a more distal radial zone than that of $\mathrm{R} 4$ neurons, in lines c819/c547 and c42 (shown for c42 in Fig. 8). As expected, comparisons of stained cell bodies in these tissues also revealed largely non-overlapping populations; at most, only four cells were double labeled. No single pattern encompassed all R4 neurons, suggesting a genetic subdivision within the R4 category into R4 distal (R4d)-type represented by pWF22-6 and c232/c507 patterns, and R4 medial (R4m)-type represented by c819/c547 and c42 patterns.

\section{Segregation of Processes within the Itr}

$\mathrm{R}$ neuron processes make compact bush-like arbors in the ltr. Golgi staining revealed the morphology to be spiny rather than bleb-like; hence, $\mathrm{R}$ neurons are presumed to receive afferent inputs at the ltr (Hanesch et al., 1989). We compared the ltr processes between different $\mathrm{R}$ neuron types. The ltr processes revealed by line c232/c507 consisted of two tufts, only one of which was double labeled in the pWF22-6 background [Fig. 9(A)], suggesting that the ltr processes of R4 and R3 type neurons are spatially segregated. Using lines c547 [Fig. 9(B)], c105 [Fig. 9(C)], and line $189 \mathrm{Y}$ (not shown) to mark the other $\mathrm{R}$ neuron types revealed that each had a distinct arbor in the ltr. The R1 type represented by c105/c561 showed considerably less tufting than the other types. In addition, this line labeled a long descending process seen arising from the ltr that has not been previously described for R1 neurons [Fig. 9(D)].

\section{Central Complex Development}

Unlike the cc of Tenebrio (Wegerhoff and Breidbach, 1992; Wegerhoff et al., 1996) and locust (Boyan and Williams, 1997), the cc in Drosophila is primarily an imaginal structure. Developmental studies of embryonic and larval pioneer tracts in Drosophila have not conclusively identified a larval counterpart or precursor of the cc (Nassif et al., 1998). However, the pb is present at the beginning of the wandering larval stage, as demonstrated by staining with antibodies to the dFMRFamide neuropeptide precursor (Schneider et al., 1993b). Beyond this, little is known about cc elaboration in Drosophila. We examined the eb enhancer-trap lines from the third-instar larval stage

Figure 6 Enhancer-trap patterns which display a single type of $\mathrm{R}$ neuron. These examples illustrate whole-mount confocal microscopy of adult brains double labeled for different enahancertrap expression patterns. (A,C) Frontal perspectives that represent reconstructions of $\sim 50 \mu \mathrm{m}$ thickness. (B,D) Approximately 5- $\mu \mathrm{m}$ cross-sections reconstructioned from (A,C), respectively, such that anterior is to the top of the figure. (A) c105 axons (green) mark the inner zone of the eb, while pwF22-6 axons (red) mark its outer zone, consistent with the R1 and R4 designations, respectively. (B) In cross-section, R4 neurons are restricted to the anterior layer, while R1 neurons arborize in the posterior layer. (C) 189y axons (green) occupy inner and medial zones of the eb and do not overlap with R4d axons (red) in the outer zone. (D) In cross-section, the majority of the $189 y$-labeled axons arborize in the anterior layer of the eb consistent with an R3 designation; some, however, also extend to the posterior layer (D). The 189y axons (D) do not progress as far posterior as do c105 axons (B). For all images, the scale bar $=20 \mu \mathrm{m}$.

Figure 7 Enhancer-trap expression patterns which display multiple types of $\mathrm{R}$ neurons. These examples illustrate whole-mount confocal microscopy of adult brains double labeled for different enahancer-trap patterns. (A) c232 (green) and pwF22-6 (red) double labeled the R4 axons (A) and cell bodies (B). c232 also stained R3 axons, similar to those labeled by 189Y [compare Fig. 7(A) green to Fig. $6(\mathrm{C})$ ]. Although they are double labeled, the entering axons appear red in (A) because intensity of the green channel was lowered to favor resolution of detail in the ring. (C) c819 (green) labeled R2- and R4-type neurons. Axons of R2- and R4-type neurons both arborize in the outer zone of the eb. Axons of R2-type neurons enter the neuropil at the eb canal (open arrow), while axons of R4 type neurons enter the neuropil from its perimeter (solid arrow). The scale bar $=20 \mu \mathrm{m}$ for all images. 


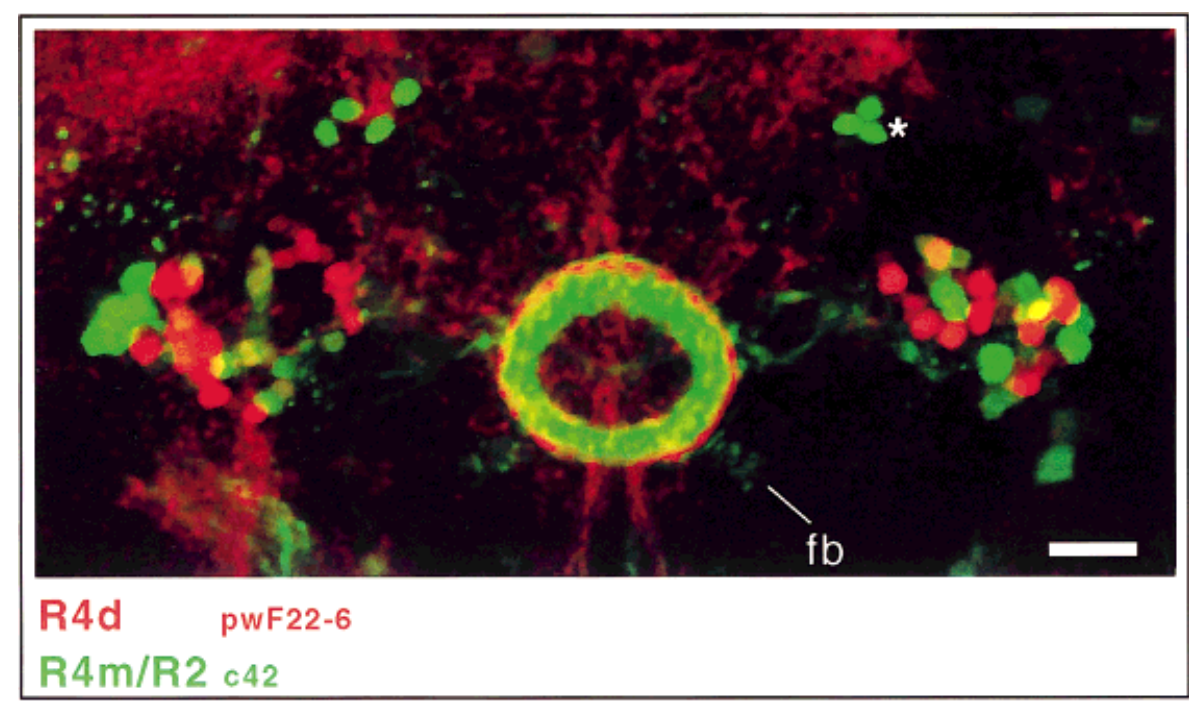

Figure 8
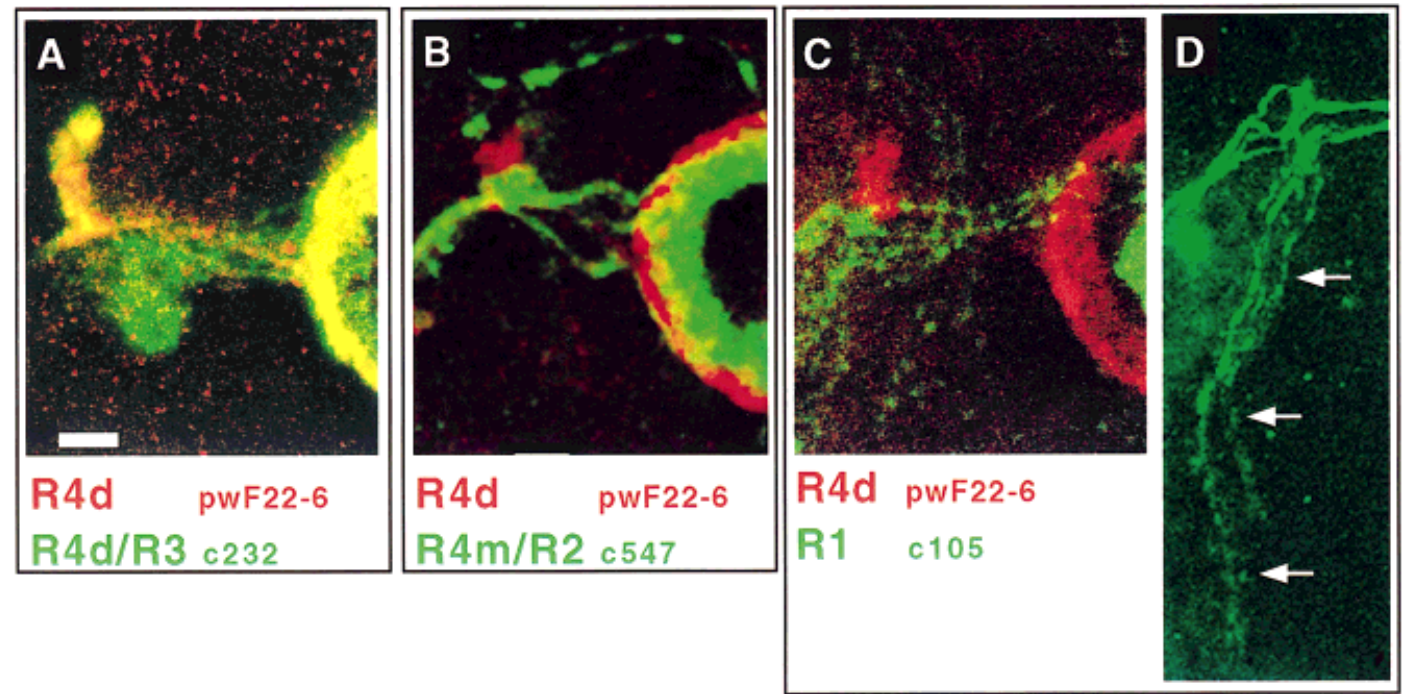

Figure 9

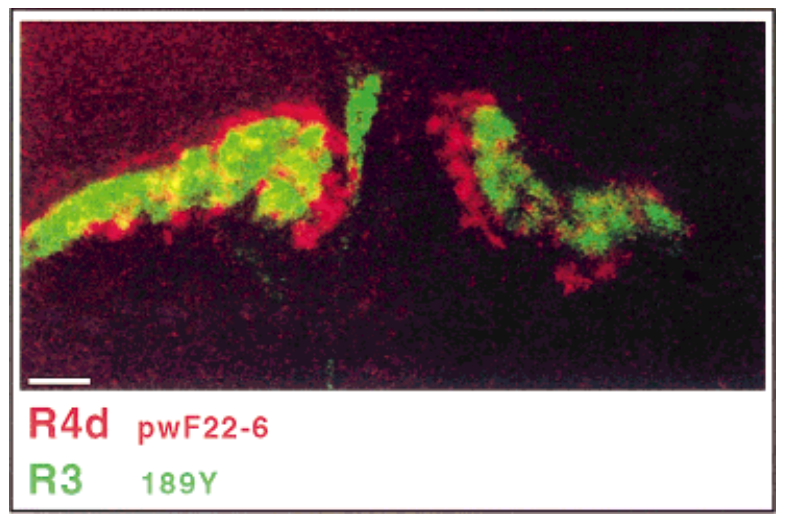

Figure 13 Confocal reconstruction of a $c b d$ mutant brain stained in whole mount for two classes of eb R-neurons. R3 axons of 189Y (green) and R4d axons of pWF22-6 (red) both show periodicity of staining, suggesting the maintenance of glomerular structure. The maintenance of neighbor relations is demonstrated by the inner position of R3 axons relative to that of the $\mathrm{R} 4 \mathrm{~d}$ axons within the otherwise disrupted eb neuropil structure. The scale bar $=10 \mu \mathrm{m}$. 
$32 \mathrm{hr}$ APF

$36 \mathrm{hr}$ APF

$48 \mathrm{hr}$ APF

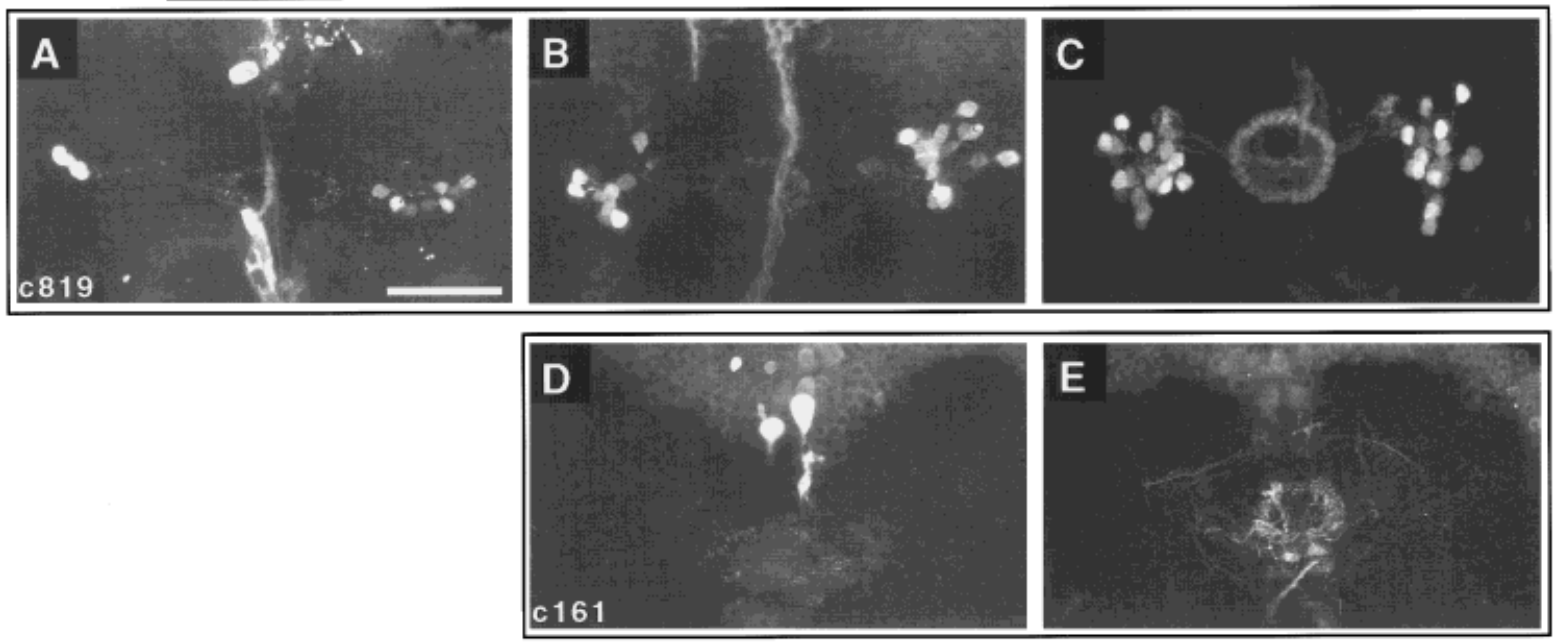

Figure 10 Development of the eb. $\mathrm{R}$ neuron axons first begin to form a ring-like neuropil recognizable at $\sim 32 \mathrm{~h} \mathrm{APF}$, as shown for $\mathrm{R} 4 \mathrm{~m}$ and $\mathrm{R} 2$ neurons of line c819 (A) The number of neurons and the eb staining intensity increases (B), and the eb structure appears nearly complete by $\sim 48 \mathrm{~h}$ APF (C). The small field eb neurons, shown for c161, also form a ring-like neuropil recognizable at $\sim 36 \mathrm{~h} \mathrm{APF} \mathrm{(D)} \mathrm{that} \mathrm{appears} \mathrm{adultlike} \mathrm{at} \sim 48 \mathrm{~h}$ APF (E). The scale bar $=50 \mu \mathrm{m}$ for all images.

through metamorphosis to ask when differentiation occurs and when the earliest vestiges of this neuropil were manifest (Fig. 10). R neuron cell bodies could be first identified in line pWF22-6 within 1-2 h of pupariation (not shown), demonstrating that at least some of the $\mathrm{R}$ neurons begin differentiation of this adult property at a very early pupal stage. Among the other eb lines, $\mathrm{R}$ neurons were first identified as a small cluster at approximately $12 \mathrm{~h}$ APF. They may have been present earlier, but were not distinguishable among other cell bodies. The characteristic ring-like eb neuropil of R-type neurons (e.g., c819) as well as of small-field neurons (e.g., c161) was first recognizable at 24-32 h [Fig. 10(A,D)] and continued to elaborate until it appeared complete by $48 \mathrm{~h}$ APF [Fig. $10(\mathrm{C}, \mathrm{E})]$. The intensity of neuropil staining and $\mathrm{R}$ neuron cell number increased throughout the remainder of adult development.

Figure 8 Enhancer-trap patterns which demonstrate the subdivision of the R4-type eb category into R4m and R4d types. This frontal perspective represents a confocal reconstruction of $\sim 50 \mu \mathrm{m}$. Some $\mathrm{c} 42$ axons (green) enter the neuropil at the perimeter and arborize in a distal zone consistent with the R4 designation. However, in eight specimens examnined, no more than four $\mathrm{c} 42$ cell bodies (green) were double labeled by another R4 enhancer pattern, pWF22-6 (red), indicating that they represent distinct R4 types. R4d axons arborize in the most distal eb zone, while R4m occupy a more medial zone. Fan-shaped neuron cell bodies (asterisk) which project to the fb are also labeled by $\mathrm{c} 42$. The scale bar $=20 \mu \mathrm{m}$.

Figure 9 Enhancer patterns that demonstrate segregation of $\mathrm{R}$ neuron processes within the ltr. These frontal perspectives represent confocal reconstructions of $\sim 15 \mu \mathrm{m}$ through the 1 tr. In all images R4d-type neurons are indicated by pWF22-6. (A) The R3 processes of c232 (green) were segregated from the R4d processes that appear yellow owing to double labeling by both c232 and pwF22-6. (B) R2- and R4m-type processes of c819 (green) are segregated from the R4d processes (red). (C) R1-type processes of c105 (green) are segregated from R4d type processes (red). (D) A single-labeled c105 tissue reveals a novel ventral projection (arrows) from the R1 ltr processes. The scale bar $=10 \mu \mathrm{m}$ for all images. 

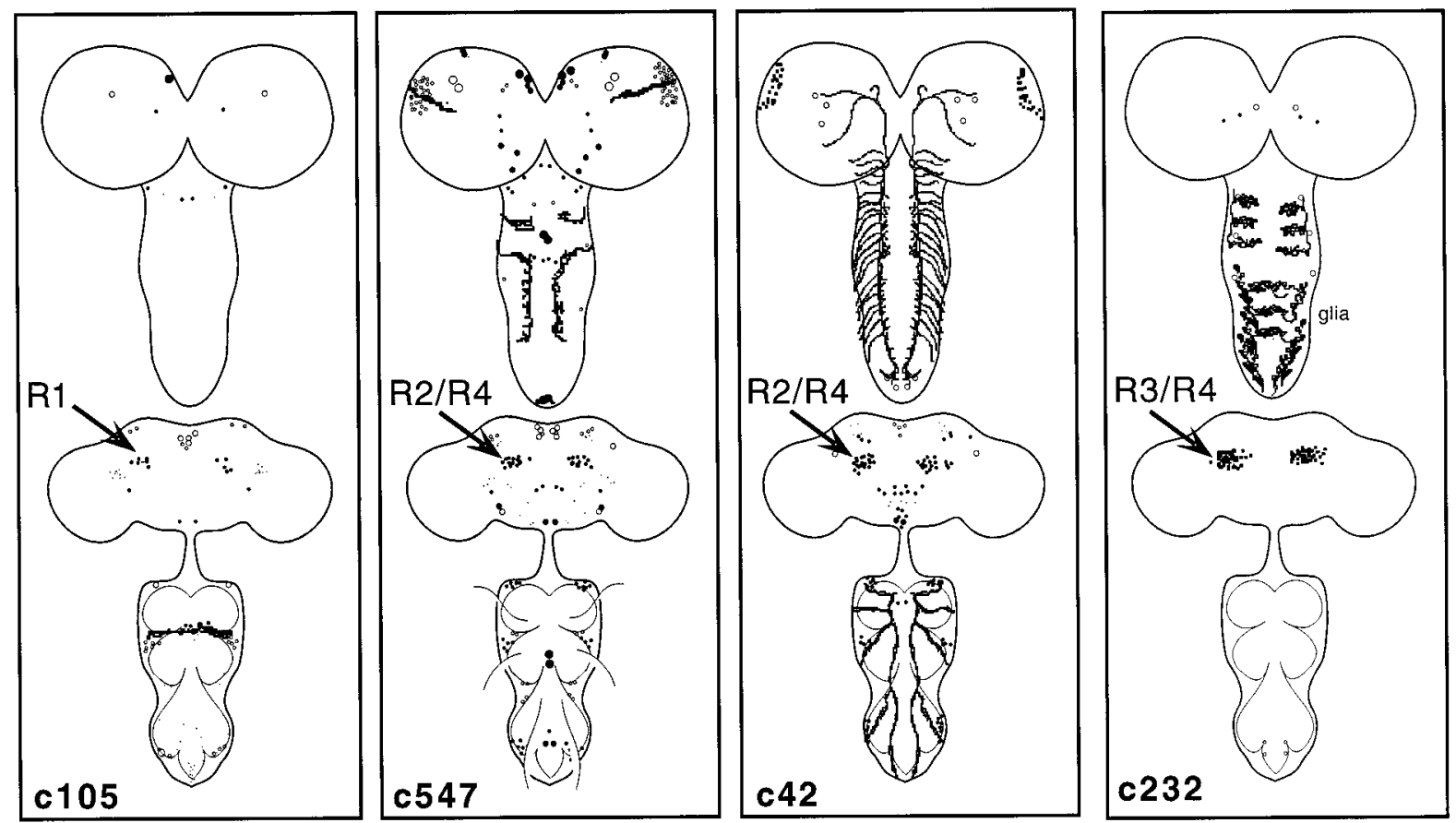

Figure 11 Schematic representation of selected cc enahancer-trap lines to illustrate the staining patterns within the CNS at both larval and adult stages. The larval information derives from histochemical stains of wandering third-instar larvae, and of adults that were 4-14 days of age of either sex. The diagrams represent the relative positions and approximate numbers of stained cells. Among the lines examined, there was no obvious correlation between staining of the $\mathrm{cc}$ and staining elsewhere in the CNS. See the text for a summary of the identifiable cell types.

\section{The P\{GAL4\} Expression Patterns in Other Cells and Tissues}

Each of the eb patterns also showed expression outside the central complex and in nonneuronal tissues. There were no pattern elements found in common throughout the collection of enhancer-trap lines we studied (Fig. 11). Even among lines with similar cc expression patterns, we could not identify other consistent pattern elements in non-cc neuropils. Several lines stained cells of the pars intercerebralis (c819/ $\mathrm{c} 547,189 \mathrm{Y}, \mathrm{c} 005$, and $\mathrm{c} 42$ ) or at the ventral midline of the segmental nerve cord (189Y and c819/c547). Line c819/c547 stained Bolwig's nerve in the larva similar to line $\mathrm{c} 161$. Line $\mathrm{c} 161$ has been previously described for its expression in proprioceptive neurons
(Shepherd and Smith, 1996). Interestingly, line pWF22-6 labeled a subset of mb Kenyon cells in the larva and early pupa. Two of the eb P\{GAL4\} lines (189Y and c115) also displayed mb expression in the adult (Armstrong et al., 1998).

\section{Mutant Analysis}

Strauss and Heisenberg (1993) described the CX mutant series: several first chromosome EMS alleles that result in characteristic deformities of the cc. We reexamined the CX mutations in the context of several eb enhancer-trap lines specific for the $\mathrm{R}$ neurons. Table 2 lists the P elements used to study R2, R3, $\mathrm{R} 4 \mathrm{~m}$, and R4d neurons in specific CX mutant back-

Table 2 Five P-Element Lines Were Used to Analyze Six CX Mutant Phenotypes

\begin{tabular}{|c|c|c|c|c|c|c|}
\hline & $\mathrm{mbd}$ & cex & ebo & ceb & $\mathrm{ccb}$ & cbd \\
\hline pWF22-6 (R4d) & & & & $\mathrm{X}$ & $\mathrm{X}$ & $\mathrm{X}$ \\
\hline c232/c507 (R4d, R3) & & $\mathrm{X}$ & $\mathrm{X}$ & $\mathrm{X}$ & & $\mathrm{X}$ \\
\hline 189Y (R3) & & & & & $\mathrm{X}$ & $\mathrm{X}$ \\
\hline $\mathrm{c} 547 / \mathrm{c} 819(\mathrm{R} 4 \mathrm{~m}, \mathrm{R} 2)$ & $\mathrm{X}$ & $\mathrm{X}$ & $\mathrm{X}$ & & & $\mathrm{X}$ \\
\hline
\end{tabular}




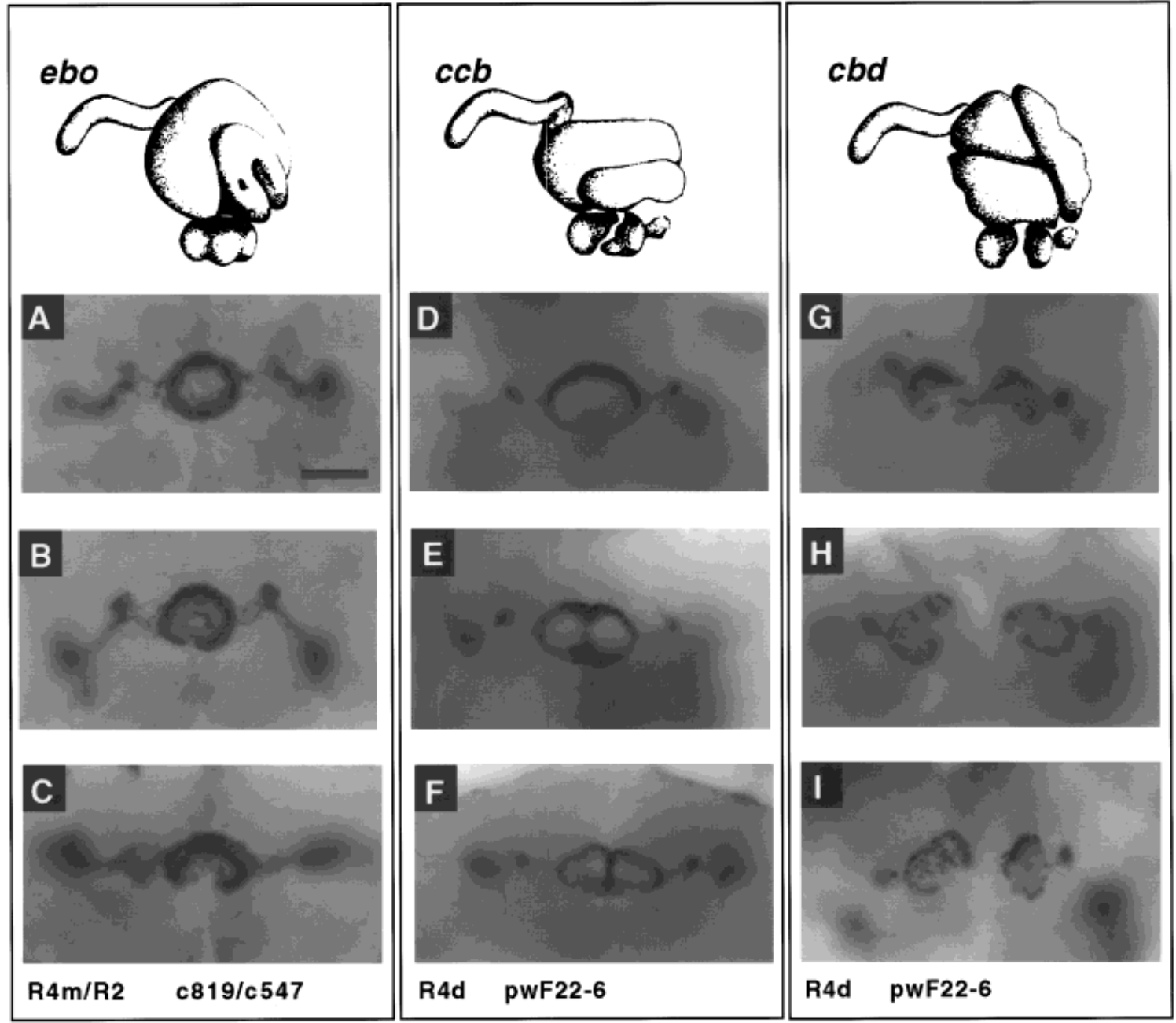

Figure 12 Analysis of eb R neuron structure in three different central complex structural mutants. Three examples of adult brains from a single eb enhancer-trap line are shown for each mutant background. The ebo mutant phenotype is illustrated by c547 (R4m and $\mathrm{R} 2$ types). The ebo mutation causes a range of phenotypes, from nearly normal (A) to completely open at the ventral aspect (C). (D-F) The $c c b$ mutant phenotype is illustrated by pWF22-6 (R4d type). The $c c b$ mutation causes a more severe range of phenotypes, from the failure to close at the ventral aspect (D) to a complete duplication of the structure (E,F). (G-I) The $c b d$ mutant phenotype is also illustrated by pWF22-6 (R4d type). The $c b d$ mutation causes the most severe range of phenotypes. Axons from either hemisphere fail to join at the midline and never form a circular structure. In all mutant backgrounds, and for all $\mathrm{R}$ neuron types examined, the location and number of cell bodies appear equivalent to wild type. The eb enhancer-trap neurons were immunostained in wholemount for $\beta$-gal and processed for the HRP-DAB reaction. The scale bar in $(\mathrm{A})=50 \mu \mathrm{m}$ for all images. The drawing atop each column is reproduced with permission from Strauss and Heisenberg (1993).

grounds. We saw a range of axonal phenotypes that were consistent with previous descriptions of the gross morphology of the deformed cc. These phenotypes ranged from mild, with just the ventral aspect of eb opened, through more severe, with a double eb, and, in the most severe cases, failure of either half to reach the midline and failure to circularize. These phenotypes were not seen in the mbd (mushroom bodies deformed) mutants which disrupt the mushroom body neuropil (data not shown). We did not assay for the R1 neuronal phenotype in these recessive X-linked mutations because our R1-marking $\mathrm{P}$ elements were also $\mathrm{X}$-linked.

Figure 12(A-C) presents examples of R2 and R4m 
neurons that illustrate the range of phenotypes observed in the ebo background (also for cex, not shown). In this background, the eb ring was sometimes not complete along its ventral aspect [Fig. 12(F)]. Figure 12(D-F) presents examples of R4d neurons that illustrate mild to severe phenotypes in the $c c b$ background (similar to $c e b$; not shown). These mutants sometimes produced a ventral eb open phenotype [Fig. 12(D)], as seen in ebo mutants; however, they also displayed a dramatic twinned-eb ("goggles") structure [Fig. 12(E,F)]. The disposition of neuronal elements in both circles was judged to be relatively normal, as evidenced by the superficial arborization of R4d neurons in each twinned eb. Figure $12(\mathrm{G}, \mathrm{I})$ presents examples of $\mathrm{R} 4 \mathrm{~d}$ neurons that illustrate the severe phenotypes in the $c b d$ background. $c b d$ animals displayed a dramatic derangement of the cc that included a failure of neuropils from the two hemispheres to fuse at the midline. On either side of the midline, axons formed eb-like structures which suggested circles, but which varied widely in shape. In most individuals, the R4d axons remained within the neuropil mass on the ipsilateral side, but in occasional animals, axons appeared to cross the midline [Fig. $12(\mathrm{G})]$.

Figure 13 presents a double-labeled $c b d$ brain that stained the R3 neurons and the R4d neurons. A high degree of organization was maintained even in this most severe phenotype. First, some glomerular structure was maintained, as indicated by the patchy appearance of the axonal terminals. Second, the relative concentric organization of $\mathrm{R}$ neuron arbors was maintained; R4d axons surrounded the R3 axons and demarcated an outer perimeter zone within a structure that otherwise poorly resembled a normal eb.

\section{DISCUSSION}

Enhancer-trap methods have proven useful in visualizing the morphology of substructures within complex neuropil such as the mbs at high resolution (Yang et al., 1995; Han et al., 1996). Here, we have applied these methods to address the organization of the $\mathrm{cc}$. From the screen of $\sim 1400$ P $\{$ GAL4 $\}$ lines, we chose 15 that stain the eb. (http//:brainbox.gla.ac.uk/flytrap presents images of lines selected from the P $\{$ GAL4 $\}$ enhancer screen for staining in other cc neuropil substructures.) The eb neurons represented by an individual enhancer expression pattern displayed similar morphology. Furthermore, we were able to correlate some of the eb staining patterns with neuronal morphologies previously described by the Golgi impregnation method (Hanesch et al., 1989). When taken into consideration with previous studies on mb neurons, such a correlation between Golgi and enhancertrap studies suggests that the subsets of neurons defined by common morphology also share gene expression characteristics. Our hypotheses are limited by the process with which the lines were selected. Because the enhancer-trap technique is most useful in identifying restricted classes of neurons, we did not examine those lines in which cc neurons were stained along with large numbers of non-cc neurons.

One particular advantage of the enhancer-trap technique as an anatomical tool is the ability to observe and compare entire classes of neurons sharing transcriptional mechanisms. There are two other existing bodies of information concerning the neuroarchitecture cc neuropil. While Golgi techniques reveal individual neurons, immunohistochemical identification of neurotransmitters in the cc neuropil reveals patterns more similar to enhancer-trap techniques. Anti-transmitter staining patterns often resemble specific elements of the neuropil subunits or specific cell types identified, and furthermore suggest a prominent role for several neuropeptides as well as classical transmitters within the cc (e.g., Homberg 1991; Vitzthum and Homberg, 1998; Schoofs et al., 1996; Vitzthum et al., 1996; Lundquist et al., 1994). Few data are available for transmitters in specific Drosophila neurons (Hanesch et al., 1989; Bouhouche et al., 1993); therefore, we have limited our comparison to Golgi classification. Our efforts to compare eb enhancer patterns with eb Golgi stains were sometimes problematic owing to differences in the two techniques. While the Golgi method clearly resolves individual fiber profiles, the enhancer-trap expression patterns typically revealed clusters of neurons with similar, grouped trajectories. Even using fluorescent immunohistochemistry and confocal microscopy, resolution of single fibers was rarely possible with enhancer-trap expression patterns. Thus, interpreting the morphology of neuronal types in enhancer lines often proved difficult. Our correlations with Golgi-derived results are largely based on the examination of prominently stained neuropil features. As a result, we may have underestimated the contributions of individual neurons (especially those occurring in small number). While these limitations are significant, they do not prevent a productive comparison of the two sets of data.

Examples of both small field and large field eb neurons were seen. Four lines displayed expression in what appeared to be groups of small-field neurons of the cc (Fig. 2). However, we could not conclusively identify specific small-field neuron types, nor could we construct a general inference regarding their ge- 


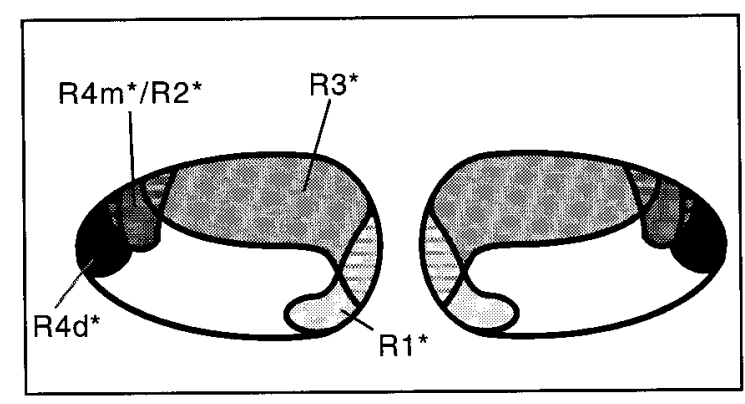

Figure 14 Schematic drawing of arborization zones observed by enhancer-trap staining. The diagram shows a coronal section of eb such that anterior is at the top of the drawing. These zones differ from those inferred by Golgi staining (Hanesch et al., 1989) because they are defined by the neuropil region occupied by all axons of a specific enahancer-trap line. The zones are designated with an asterisk $(\mathrm{R} \# *)$ to emphasize that the interpretations are based on different techniques. In addition to the prominent R-type axons, the indicated zones may include processes of other cell types.

netic classifications from these patterns. Hence, the degree to which cohorts of small-field cc neurons reflect separate transcriptional mechanisms is still unresolved. The remaining $11 \mathrm{eb}$ lines stained large-field $\mathrm{R}$ neurons, several of which could be correlated with Golgi described morphology. Figure 14 summarizes the four arborization zones in the eb defined by confocal analysis of enhancer-trap lines that represented each type of $\mathrm{R}$ neuron. Because of the different natures of Golgi and enhancer-trap analysis, these zones differ slightly from those described by Hanesch et al. (1989). In addition to the prominent R-type axons, the indicated zones may include processes of additional cell types included in the enhancer-trap pattern.

The use of the pWF22-6 lacZ line as a counterstain for GAL4-driven gene expression permitted us a detailed examination of the R4 category of eb R neurons. Hanesch et al. (1989) described the R4 category but did not rule out the possibility of heterogeneity. Our results show that the R4 neuron category is divisible into neurons whose axons arborize in the distal zone (R4d: e.g., pWF22-6 and c232/c507) and those that arborize in an immediately adjacent, medial domain (R4m: e.g., c819/c547 and c42). Further evidence for the hypothesis of multiple R4 cell types derives from the fact that among R4d patterns, not all neurons double stained [Fig. 9(B)]. This subdivision of morphologically similar cells by distinct enhancer expression is likely to be prototypic for neurons in other areas of the cc. Our analysis took advantage of the different natures of Golgi and enhancer-trap tech- niques. We used the relationship between the two techniques to increase our comprehension of cc structure. Because of the stochastic and random nature of Golgi staining, patterns could not be identified, but only inferred. In contrast, enhancer-trap staining revealed patterns while individual cell morphology had to be inferred. These techniques have been similarly applied to study the mushroom body neuropil (Yang et al., 1995). For the mushroom bodies, detailed classification of neuronal pathways was not possible based on Golgi staining alone. Enhancer-traps lines revealed morphological differences which provided the search images such that mb cell trajectories could be recognized among the Golgi data (Yang et al., 1995).

Our ability to compare specific classes of R neurons allowed us to ask whether their ltr processes exhibited spatial segregation comparable to that observed for their eb processes. The spiny, ltr processes of $\mathrm{R}$ neurons are presumed to receive afferent input, from the optic foci, and from fibers of widely branching neurons in the protocerebrum (Hanesch et al., 1989). Hanesch et al. (1989) noted that R neuron ltr processes displayed exceptionally compact bushes. We found a significant degree of segregation among ltr processes of the R2-, R3-, R4m-, and R4d-type neurons (Fig. 9). We therefore propose that large-field neurons of the eb exhibit topographic segregation of both afferent and efferent branches.

We did not find evidence for consistent spatial arrangement of $\mathrm{R}$ neurons at the level of the neuronal cell bodies. Spatial segregation of neuronal somata according to enhancer-trap expression has been demonstrated for the Kenyon cells of the mb (Yang et al., 1995; Ito et al., 1997). The Kenyon cells arise from four neuroblasts which form a quadruple structure of clonal units, each a product of a single neuroblast (Ito et al., 1997; Armstrong et al., 1998). The segregation is therefore first a product of lineage, and is then restricted by cell type within the individual unit. Although four dorsal neuroblasts are known to contribute a majority of $\mathrm{cc}$ neurons projecting to the $\mathrm{pb}$ and $\mathrm{fb}$ substructures in the locust (Boyan and Williams, 1997), little is known about the lineage of most specific cc neuronal types including the $\mathrm{R}$ neurons. The collection of enhancer-trap lines presented here will prove useful in further studies of this nature.

Although we observed CNS staining at all postembryonic stages, few recurring features were identified. mb staining was seen in two of the eb P $\{$ GAL4 $\}$ lines (189Y and c115) as well as in larval pWF22-6 brain (S. C. P. Renn, unpublished observations). The nature of these expression patterns may reveal a moleculargenetic link between certain subsets of neurons in the 
$\mathrm{mb}$ and eb. As these are both higher-order neuropils, these preliminary indications of morphological and/or genetic connections suggest that further analysis of this correlations will be useful.

Enhancer-trap expression patterns prior to adulthood revealed features of eb development. The observations are consistent with the view that in Drosophila the $\mathrm{cc}$ is essentially an adult-specific brain structure, with only a rudimentary counterpart in the larval brain. Initial expression of enhancer activity occurred within a small number of neurons prior to $24 \mathrm{~h} \mathrm{APF}$, the first axons began to form a recognizable ring by $32 \mathrm{~h}$ APF, and cell number and axon density increased throughout the remainder of adult development. These observations support the hypothesis that small collections of cells and cellular processes serve as a scaffold for later-growing neurons. The eb was not prefigured by any single type of $\mathrm{R}$ neuron or small-field neuron that we studied. Rather, each pattern emerged as a subset of the final staining pattern. In orthopteran insects, the cc is fully formed during embryogenesis beginning with axogenesis at 55\% of embryogenesis (Boyan and Williams, 1997). In the holometabolous beetle Tenebrio, eb development is the last step of cc development: It occurs at the start of metamorphosis after the other cc structures have developed during late larval stages (Wegerhoff and Breidbach, 1992).

Ethylmethane sulphonate-induced single gene mutations that disrupt cc structure in the CX lines have been used to address cc functions. Each CX mutant shows a characteristic set of behavioral deficits such as poor phototaxis, reduced walking speed, and abnormal object fixation. At least at a gross level, the anatomical deficits appear limited to the cc, and given their behavioral correlates, a role for the $\mathrm{cc}$ in motor coordination has been proposed (Strauss and Heisenberg, 1993). The genes affected by these mutations have not yet been identified; however, our phenotypic analysis allows us to asses which developmental processes are or are not affected.

The process of neuroblast proliferation is disrupted by two mutations affecting other neuropil structures: either overproduction in the case of mushroom body defect (Prokop and Technau, 1994) or underproduction in the case of minibrain (Tejedor et al., 1995). Our analysis of eb enhancer lines in the CX mutants indicates that the gross structural distortions did not result from the failure to produce either the normal number or normal diversity of $\mathrm{R}$ neurons. Two different processes of axon growth also occurred normally in the CX mutants. Proper directional growth toward the midline and elaboration of appropriately segregated axon terminals within the eb appeared to proceed normally. The long-range guidance and maintenance of neighbor relations may be intimately connected, or may reflect different mechanisms of cell recognition. Even when displaced to the greatest extents in the most severe phenotypic cases ( $c b d$ animals), both R3 and R4 axons maintained proper neighbor relations. Furthermore, the axons were capable of forming aggregations of clustered terminals with a strong suggestion of glomerular formation (Figs. 12 and 13).

The processes that were affected in the CX mutants involved the final steps of ring formation. There was a correlation between final distance from the midline, and the ability to complete a ringlike structure. The axons in $c c b$ and $c e b$ lines typically reached the midline and successfully participated in formation of a ring-shaped neuropil even when the axons failed to interweave their terminal arbors with those of contralateral homologues [Fig. 12(F)]. Such neuropils were twinned and occupied immediately adjacent domains on either side of the midline. In contrast, the $c b d$ mutation prevented ring formation and the axons failed to reach (or avoided) the midline. The requirement for the $c b d$ gene may be autonomous to the $\mathrm{R}$ neurons, or may be a nonautonomous effect due to their failure to reach the midline, where they normally receive ring-promoting cues. At some level, the process of ring formation must require the participation of specific gene products, but it may also be facilitated by mechanical constraints that favor its particular geometry (Van Essen, 1997; Condron and Zinn, 1997). The actual steps involved in these processes can be studied when more is known about the normal growth of the $\mathrm{R}$ axons and when the genes perturbed in the CX mutant series are studied. In the ventral nerve cord, the genes comm and robo promote antagonistic functions in directing the decussation of a correct number of axons (Kidd et al., 1996; Tear et al., 1996; Seeger et al., 1993). Using these P\{GAL4\} enhancer-trap lines to misexpress molecules known to participate in axon guidance and/or synapse formation (Tessier-Lavigne and Goodman, 1996) may be a useful alternate strategy in this regard.

Detailed analysis of enhancer-trap expression patterns complements traditional anatomical methods in the evaluation of the structure and function of brain regions. This collection of eb enhancer-trap lines indicates a potential for defining distinct cohorts of neurons that underlie the behavioral roles of the $\mathrm{cc}$ both from a morphological and a molecular perspective. Expansion of this collection to include enhancertrap lines which indicate projections to and from the $\mathrm{cc}$ will also be useful for understanding the function of the cc. P element-mediated enhancer-trap tech- 
niques reveal genomic elements that direct expression to a subset of cells: Such elements may normally be used to direct expression of specific genes in these patterns (e.g., Jones et al., 1995; Cheyette et al., 1994). The identification of genes tagged by these inserts might be of immediate value. To date, two of the insertions here studied have been analyzed at the molecular level. Osborn et al. (1997) reported that the $189 \mathrm{Y}$ P-element localizes to the for locus and is inserted within a gene encoding a cGMP-dependent protein kinase. This gene mutates to display behavioral alterations in larval foraging. It is not yet known where the for gene product is required. The staining pattern of c232/c507 appears identical to that of an alkaline phosphatase gene adjacent to the P-element insertion (Yang, unpublished observations). The $\mathrm{P}\{\mathrm{GAL} 4\}$ enhancer-trap system offers the particular advantage of driving expression agents to alter or ablate cell function (O'Dell et al., 1995; McNabb et al., 1997) to analyze neural function and behavioral affects. Similar methods of gene identification and disruption will be useful to test specific hypotheses concerning the role of the ellipsoid body in behavior.

The authors thank Martin Heisenberg for CX mutants as well as for ideas and discussion from the start of this project. The following people generously contributed additional fly stocks: Mei-li Joiner, Klemens Stoertkuhl, Karl Fischbach, David Shepard, Jochim Urban, Gabrielle Boulianne, Z. Nie, Sean Sweeney, and Cahir O'Kane. Also, the authors thank Heiner Niemann for the anti-TeTxLC antibody, and Jeff Lichtman and Mehmet Dokocu for assistance with the confocal microscope and image software. This work was supported by grants from the UK BBSRC and the Wellcome Trust, to KK, and a grant from the National Institutes of Health (NS-21749) to PHT.

\section{REFERENCES}

Armstrong JD, deBelle JS, Wang Z, Kaiser K. 1998. Metamorphosis of the mushroom bodies: large scale rearrangements of the neural substrates for associative learning and memory in Drosophila. Learn Mem 5:102-114.

Armstrong JD, Kaiser K. 1997. The study of Drosophila brain development. In: Houdebine LM, editor. Transgenic animals: generation and use. Harwood. p 365-370.

Bausenwein B, Müller NR, Heisenberg M. 1994. Behaviordependent activity labeling in the central complex of the Drosophila during controlled visual learning. J Comp Neurol 340:455-468.

Bellen HJ, O'Kane CJ, Wilson C, Grossniklaus U, Pearson RK, Gehring WJ. 1989. P-element-mediated enhancer detection: a versatile method to study development in Drosophila. Genes Dev 3:1288-1300.

Bier E, Vaessin H, Shepherd S, Lee K, McCall K, Barbel S,
Ackerman L, Carretto R, Uemura T, Grell E. 1989. Searching for pattern and mutation in the Drosophila genome with a P-lacZ vector. Genes Dev 3:1273-1287.

Bouhouche A, Vaysse G, Corbiere M. 1993. Immunocytochemical and learning studies of a Drosophila melanogaster neurological mutant, no-bridge as an approach to the possible role of the central complex. J Neurogenet 9:105-141.

Boyan GS, Williams JLD. 1997. Embryonic development of the pars intercerebralis/central complex of the grasshopper. Dev Genes Evol 207:317-329.

Brand A, Perrimon N. 1993. Targeted gene expression as a means of altering cell fates and generating dominant phenotypes. Development 118:401-415.

Brand AH, Dormand EL. 1995. The GAL4 system as a tool for unraveling the mysteries of the Drosophila nervous system. Curr Opin Neurobiol 5:574-578.

Cheyette BN, Green PJ, Martin K, Garren H, Hartenstein V, Zipursky SL. 1994. The Drosophila sine oculis locus encodes a homeodomain-containing protein required for the development of the entire visual system. Neuron 12:977-996.

Condron BG, Zinn K. 1997. Regulated neurite tension as a mechanism for determination of neuronal arbor geometries in vivo. Curr Biol 7:813-816.

Graham D. 1979a. Effects of circum-oesophageal lesion on the behavior of the stick insect Carausius morosus: II. Changes in walking co-ordination. Biol Cybernet 34: $147-154$

Graham D. 1979b. Effects of circum-oesophageal lesion on the behavior of the stick insect Carausius morosus: I. Cyclic behavior patterns. Biol Cybernet 34:139-145.

Han PL, Meller V, Davis RL. 1996. The Drosophila brain revisited by enhancer detection. J Neurobiol 31:88-102.

Hanesch U, Fischbach KF, Heisenberg M. 1989. Neural architecture of the central complex in Drosophila melanogaster. Cell Tissue Res 457:343-366.

Heisenberg M. 1989. Genetic approach to learning and memory (mnemogenetics) in Drosophila melanogaster. In: Rahmann B, editor. Fundamentals of memory formation: neural plasticity and brain function. Stuttgart: Gustave Fisher Verlag. p 3-45.

Heisenberg M. 1994. Central brain function in insects: genetic studies on the mushroom bodies and central complex in Drosophila. In: Schildberger K, editor. Neural basis of behavioral adaptations. Stuttgart: Gustav Fisher Verlag. p 61-79.

Heisenberg M, Borst A, Wagner S, Byers D. 1985. Drosophila mushroom body mutants are deficient in olfactory learning. J Neurogenet 4:1-30.

Homberg U. 1985. Interneurons of the central complex in the bee brain (Apis mellifera, L.). J Insect Physiol 31: 451-464.

Homberg U. 1987. Structure and function of the central complex in insects. In: Gupta AP, editor. Arthropod brain: its evolution, development, structure, and function. New York: Wiley. p 347-367.

Ilius M, Wolf R, Heisenberg M. 1994. The central complex 
of Drosophila melanogaster is involved in flight control: studies on mutants and mosaics of the gene ellipsoid body open. J Neurogenet 9:189-206.

Ito K, Awano W, Zuzuki K, Hiromi Y, Yamamoto D. 1997. The Drosophila mushroom body is a quadruple structure of clonal units each of which contains a virtually identical set of neurons and glial cells. Development 124:761-771.

Jones BW, Fetter RD, Tear G, Goodman CS. 1995. glial cells missing: a genetic switch that controls glial versus neuronal fate. Cell 82:1013-1023.

Kidd T, Brose K, Mitchell KJ, Fetter RD, Tessier-Lavigne M, Goodman CS, Tear G. 1998. roundabout controls axon crossing of the CNS midline and defines a novel subfamily of evolutionarily-conserved guidance receptors. Cell 92:205-215.

Lundquist CT, Clottens FL, Holman GM, Riehm JP, Bonkale W, Nassel DR. 1994. Locustatachykinin immunoreactivity in the blowfly central nervous system and intestine. J Comp Neurol 341:225-240.

McNabb SL, Baker JD, Agapite J, Steller H, Riddiford LM, Truman J. 1997. Disruption of a behavioral sequence by targeted death of peptidergic neurons in Drosophila. Neuron 19:813-823.

Mizunami M. 1995. Morphology of higher-order ocellar interneurons in the cockroach brain. J Comp Neurol 362: 293-304.

Muller M, Homberg U, Kuhn A. 1997. Neuroarchitcture of the lower division of the central body in the brain of the locust (Schistocerca gragaria). Cell Tissue Res 288:159-176.

Nassif C, Noveen A, Hartenstein V. 1998. Embryonic development of the Drosophila brain. I. Pattern of pioneer tracts. J Comp Neurol 420:10-31.

O’Dell KMC, Armstrong JD, Yang MY, Kaiser K. 1995. Functional dissection of the Drosophila mushroom bodies by selective feminization of genetically defined subcompartments. Neuron 15:56-61.

Osborne KA, Robichon A, Burgess E, Butland S, Shaw RA, Coulthard A, Pereira HS, Greenspan RJ, Sokolowski MB. 1997. Natural behavior polymorphism due to a cGMPdependent protein kinase of Drosophila. Science (Wash) 8:834-836.

Pardue ML. 1986. In situ hybridization to DNA of chomosomes and nuclei. In: Roberts DB, editor. Drosophila: a practical approach. Oxford: IRL Press. p 111-137.

Power ME. 1943. The brain of Drosophila melanogaster. J Morphol 74:517-559.

Prokop A, Technau GM. 1994. The normal function of the mushroom body defect gene in Drosophila and proliferation of neuroblasts. Dev Biol 161:321-337.

Schildberger K. 1983. Local interneurons associated with the mushroom bodies and the central body in the brain of Acheta domesticus. Cell Tissue Res 230:573-586.

Schneider LE, Roberts MS, Taghert PH. 1993a. Cell typespecific transcriptional regulation of the Drosophila FMRFamide neuropeptide gene Neuron 10:279-291.

Schneider LE, Sun ET, Garland DJ, Taghert PH. 1993b. An immunocytochemical study of the FMRFamide neu- ropeptide gene products in Drosophila. J Comp Neurol 337:446-460.

Schoofs L, Veelaert D, Vander Broeck J, De Loof A. 1996. Immunocytochemical distribution of locustamyoinhibiting peptide (Lom-MIP) in the nervous system of Locusta migratoria. Regul Peptide 63:171-179.

Schurmann FW, Klemm N. 1984. Serotonin-immunoreactive neurons in the brain of the honeybee. J Comp Neurol 225:570-580.

Seeger M, Tear G, Ferres-Marco D, Goodman CS. 1993. Mutations affecting growth cone guidance in Drosophila genes necessary for guidance toward and away from the midline. Neuron 10:409-426.

Shepherd D, Smith SA. 1996. Central projections of persistent larval sensory neurons prefigure adult sensory pathways in the CNS of Drosophila. Development 122:23752384.

Strausfeld NJ. 1976. Atlas of an insect brain. New York: Springer Heidelberg. p. 80-88.

Strauss R, Hanesch U, Kinkelin M, Wolf R, Heisenberg M. 1992. No-bridge of Drosophila melanogaster: portrait of a structural brain mutant of the central complex. J Neurogenet 8:145-155.

Strauss R, Heisenberg M. 1993. A higher control center of locomotor behavior in the Drosophila brain. J Neurosci 13:1854-1861.

Sweeney ST, Broadie K, Keane J, Niemann H, O'Kane CJ. 1995. Targeted expression of tetanus toxin light chain in Drosophila specifically eliminates synaptic transmission and causes behavioral defects. Neuron 4:341-351.

Tear G, Harris R, Sutaria S, Kilomanski K, Goodman CS, Seeger MA. 1996. commissureless controls growth come guidance across the CNS midline in Drosophila and encodes a novel membrane protein. Neuron 16:501-514.

Tejedor F, Zhu XR, Kaltenbach E, Ackermann A, Bausmann A, Cana I, Heisenberg M, Fischbach KF, Pongs O. 1995. minibrain: a new protein kinase family involved in postembryonic neurogenesis in Drosophila. Neuron 14: 287-301.

Tessier-Lavigne M, Goodman CS. 1996. The molecular biology of axon guidance. Science (Wash) 274:11231133.

Van Essen DC. 1997. A tension-based theory of morphogenesis and compact wiring in the central nervous system. Nature (Lond) 385:313-318.

Vitzthum H, Homberg U. 1998. Immunocytochemical demonstration of locustatachykinin-related peptides in the central comples of the locust brain. J Comp Neurol 390: 455-469.

Vitzthum H, Homberg U, Agricola H. 1996. Distribution of Dip-allatostatinI-like immunoreactivity in the brain of the locust Schistocerca gregaria with detailed analysis of immunostainging in the central complex. J Comp Neurol 369:419-437.

Wadepuhl M, Huber F. 1979. Elicitation of singing and courtship movements by electrical stimulation of the brain of the grasshopper. Naturwissenschaften 66:340-344.

Wegerhoff R, Breidbach O. 1992. Structure and development 
of the larval central complex in a holometabolous insect, the beetle Tenebrio molitor. Cell Tissue Res 268:341-358.

Wegerhoff R, Breidbach O, Lobemeier M. 1996. Development of locustatachykinin immuopositive neurons in the central complex of the beetle Tenebrio molitor. J Comp Neurol 375:157-166.

Williams JLD. 1975. Anatomical studies of the insect central nervous system: a ground-plan of the midbrain and an introduction to the central complex in the locust, Schistocerca gregaria (Orthoptera). J Zool (Lond) 176:67-86.

Yang MY, Armstrong JD, Vilinsky I, Strausfeld NJ, Kaiser K. 1995. Subdivision of the Drosophila mushroom bodies by enhancer-trap expression patterns. Neuron 15:45-54.

Yeh E, Gustafson K, Boulianne GL. 1995. Green fluorescent protein as a vital marker and reporter of gene expression in Drosophila. Proc Nat Acad Sci USA 92: 7036-7040. 